\title{
An analytical-numerical approach to simulate the dynamic behaviour of arbitrarily laminated composite plates
}

\author{
Liz G. Nallim ${ }^{\mathrm{a}, *}$, Sergio Oller ${ }^{\mathrm{b}}$ \\ ${ }^{a}$ CONICET, Facultad de Ingeniería, Universidad Nacional de Salta, Av. Bolivia 5150, 4400 Salta, Argentina \\ ${ }^{\mathrm{b}}$ Departamento de Resistencia de Materiales y Estructuras en la Ingeniería, Universidad Politécnica de Cataluña, Campus Norte UPC, \\ Gran Capitán S/N, 08034 Barcelona, Spain
}

Available online 23 October 2007

\begin{abstract}
A general analytical-numerical approach developed for the dynamical analysis of unsymmetrically laminated plates of general quadrilateral planforms is presented in this work. An arbitrary quadrilateral thin flat laminate is mapped onto a square basic one, so that a unique macro-element is constructed for the whole plate. The Ritz method is applied to evaluate the governing equation in which the coupling effects of bending and stretching are contained. All possible transverse boundary conditions combining with the different inplane constraints are considered in the analysis. The resulting algorithm possesses great flexibility, it is easy to program and it needs minimal input information. For these reasons, the proposed methodology results convenient for large scale structural design and analysis where repeated calculations are often required.
\end{abstract}

(C) 2007 Elsevier Ltd. All rights reserved.

Keywords: Laminated plates; Elasticity; Free vibration

\section{Introduction}

Fibre-reinforced composite laminates are very important in many engineering applications. In addition to their high strength/light-weight, another important advantage of composite laminates is that structural properties can be tailored through changing the fibre angle and/or the number and sequence of plies. Particularly, laminated plates of different shapes made of advanced fibre-reinforced composite materials have many excellent advantages and are widely used as high-performance structural components. The accurate and efficient determination of the natural vibration frequencies and mode shapes of laminated plates components are essentials to the design and performance evaluation of a mechanical system. Moreover, the plate resonant frequencies and vibration mode shapes are often

\footnotetext{
* Corresponding author. Tel.: +54 387 4258615; fax: +54 3874255351.

E-mail addresses: Inallim@unsa.edu.ar (L.G. Nallim), sergio.oller@ upc.edu (S. Oller).
}

used to establish the dynamic response of complex engineering systems.

General angle-ply laminated plates exhibit various coupling effects, such as stretching-bending, stretching-shear and bending-twisting couplings, due to the anisotropy of the individual lamina and unsymmetrical layering [1]. Existence of these couplings and the general quadrilateral plate formulation are the source of analytical difficulties and of complicated mathematical structures for the boundary conditions making difficult the exact analysis for even the simplest cases. Consequently, most studies on these laminates employ approximate analytical or numerical methods.

A deep revision among the references about free vibration of thin unsymmetrically laminated composite plates reveals that most papers are concerned with rectangular plates (see e.g. [2-7]). Only limited literature can be found for unsymmetrically laminated plates of general quadrilateral shapes. In this topic, for instance, Liew and Lim [8] and Lim et al. [9] investigated the free vibration of trapezoidal multi-layered laminates with different combinations of boundary conditions using two-dimensional orthogonal 
polynomials as the trial functions in the Ritz method. For the study of free vibration of skew laminates Wang [10] used B-spline Rayleigh-Ritz method, while Krishna Reddy and Palaninathan [11] used a general high precision triangular plate bending finite element.

In general, it is observed that the Rayleigh-Ritz method has had a frequent application because of its high accuracy and relatively small computational cost, mainly related with the use of only one single super element in the whole process. The fundamental difficulty associated with the Ritz method in complex laminates is the choice of suitable functions to approximate the deflected shape. In a previous paper [12] the authors developed a general algorithm based on the Ritz method in conjunction with natural coordinates to express the geometry of laminated plates of general quadrilateral shapes in a simple form. That methodology was limited to the analysis of symmetrically laminated composite plates, where only the bending-twisting coupling needed to be included. In view of the aforementioned limitation, the present work has been undertaken to extend the mentioned algorithm to embrace laminates with unsymmetrical layering. The procedure involves the reformulation of the use of the geometric natural-to-Cartesian

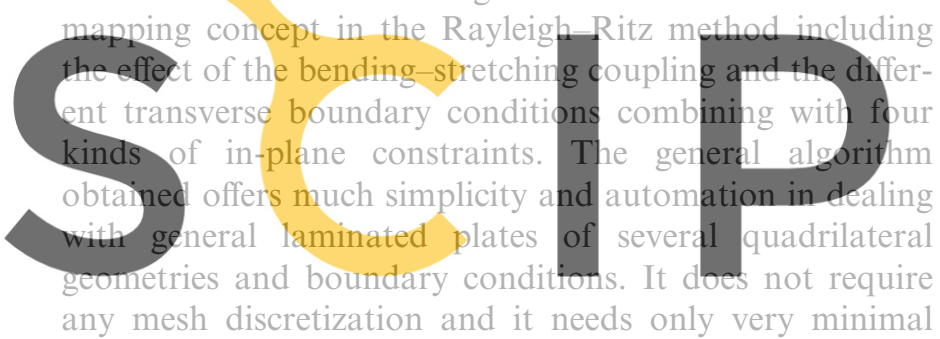

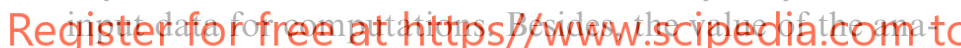
Tytical solution obtained here is that it allows getting insight into the behaviour on complex laminated plates.

To show the accuracy and the correctness of the presented method, convergence tests are carried out for several selected plate problems and, when it is possible, some results are compared with those published by other authors.

The algorithm developed can be applied to the analysis of a wide range of laminates with different shapes, aspect ratios, boundary conditions, number of layers, stacking sequences and angles of fibre orientation. The number of parameters is too high and the possibility of combination among them is infinite. For these reasons natural frequencies and nodal patterns of free vibration are presented for selected representative cases which can also be useful as benchmark comparison for future investigations in this topic.

\section{Formulation}

\subsection{Energy functional components}

Consider a flat, thin arbitrary-shaped quadrilateral fibre reinforced composite laminated plate of uniform thickness $h$ as shown in Fig. 1a. The plane $x-y$ is placed at the middle surface of the plate thickness, while $z$ remains normal to it. In each layer of the laminate $\beta$ denotes the angle of fibre orientation and the major and minor principal material axes are denoted by $L$ and $T$, respectively. In consequence, the material constants are denoted by $E_{L}, E_{T}, v_{L T}$ and $G_{L T}$.

The fundamental displacements are the three mid-surface translational displacements $u, v$ and $w$ along the $x, y$ and $z$ directions, respectively. It is necessary that the two in-plane mid-surface translational displacements $u$ and $v$ are included in the analysis due to the coupling between in-plane and out-of-plane behaviour in laminates with unsymmetrical layup. Assuming that the Kirchhoff hypothesis holds, the translational displacements $\bar{u}, \bar{v}, \bar{w}$, at a general point in the laminate are given by:

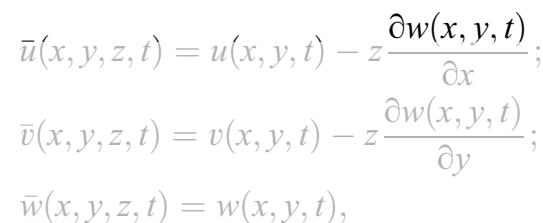

\section{where $t$ is the time dimension.}

During free vibration, the displacements are assumed split in the spatial and temporal parts, being the last one

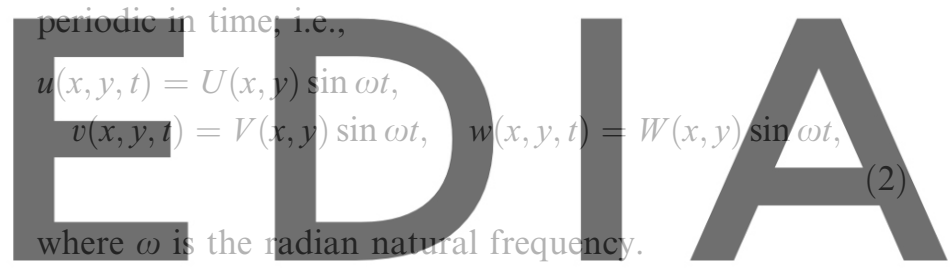

Taking into account Eq. (2), the maximum strain energy of the unsymmetrically laminated plate can be written as download the version without the watermark

$$
\begin{aligned}
& U_{\max }=\frac{1}{2} \iint_{R} L A_{11}\left(\frac{\partial U}{\partial x}\right)^{2}+2 A_{12} \frac{\partial U}{\partial x} \frac{\partial V}{\partial y}+A_{22}\left(\frac{\partial V}{\partial y}\right) \\
& +2 A_{16}\left(\frac{\partial U}{\partial x} \frac{\partial U}{\partial y}+\frac{\partial U}{\partial x} \frac{\partial V}{\partial x}\right) \\
& +2 A_{26}\left(\frac{\partial U}{\partial y} \frac{\partial V}{\partial y}+\frac{\partial V}{\partial y} \frac{\partial V}{\partial x}\right)+A_{66}\left(\frac{\partial U}{\partial y}+\frac{\partial V}{\partial x}\right)^{2} \\
& -2 B_{11} \frac{\partial U}{\partial x} \frac{\partial^{2} W}{\partial x^{2}}-2 B_{12}\left(\frac{\partial U}{\partial x} \frac{\partial^{2} W}{\partial y^{2}}+\frac{\partial V}{\partial y} \frac{\partial^{2} W}{\partial x^{2}}\right) \\
& -2 B_{22} \frac{\partial V}{\partial y} \frac{\partial^{2} W}{\partial y^{2}} \\
& -2 B_{16}\left(\frac{\partial V}{\partial x} \frac{\partial^{2} W}{\partial x^{2}}+\frac{\partial U}{\partial y} \frac{\partial^{2} W}{\partial x^{2}}+2 \frac{\partial U}{\partial x} \frac{\partial^{2} W}{\partial x \partial y}\right) \\
& -2 B_{26}\left(\frac{\partial U}{\partial y} \frac{\partial^{2} W}{\partial y^{2}}+\frac{\partial V}{\partial x} \frac{\partial^{2} W}{\partial y^{2}}+2 \frac{\partial V}{\partial y} \frac{\partial^{2} W}{\partial x \partial y}\right) \\
& -4 B_{66} \frac{\partial^{2} W}{\partial x \partial y}\left(\frac{\partial U}{\partial y}+\frac{\partial V}{\partial x}\right)+D_{11}\left(\frac{\partial^{2} W}{\partial x^{2}}\right)^{2} \\
& +2 D_{12} \frac{\partial^{2} W}{\partial x^{2}} \frac{\partial^{2} W}{\partial y^{2}}+D_{22}\left(\frac{\partial^{2} W}{\partial y^{2}}\right)^{2}+4 D_{16}\left(\frac{\partial^{2} W}{\partial x^{2}} \frac{\partial^{2} W}{\partial x \partial y}\right) \\
& \left.+4 D_{26}\left(\frac{\partial^{2} W}{\partial y^{2}} \frac{\partial^{2} W}{\partial x \partial y}\right)+4 D_{66}\left(\frac{\partial^{2} W}{\partial x \partial y}\right)^{2}\right] \mathrm{d} x \mathrm{~d} y
\end{aligned}
$$



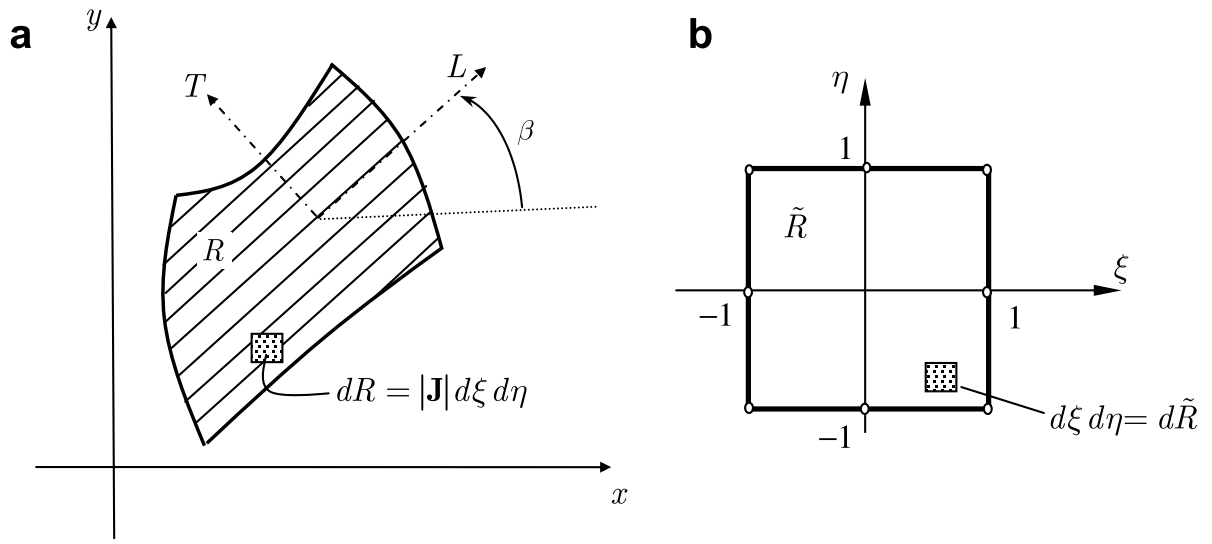

Fig. 1. (a) Geometry of a quadrilateral laminated plate in Cartesian coordinate system and (b) square parent domain in natural coordinate system.

where $A_{i j}, B_{i j}$ and $D_{i j}(i, j=1,2,6)$ denote the stretching,
stretching-bending coupling and bending stiffness coeffi-
cients, respectively $[1,13]$ and $R$ is the mid-surface area
(Fig. 1a).
The maximum kinetic energy for free vibrations of the plate is given by

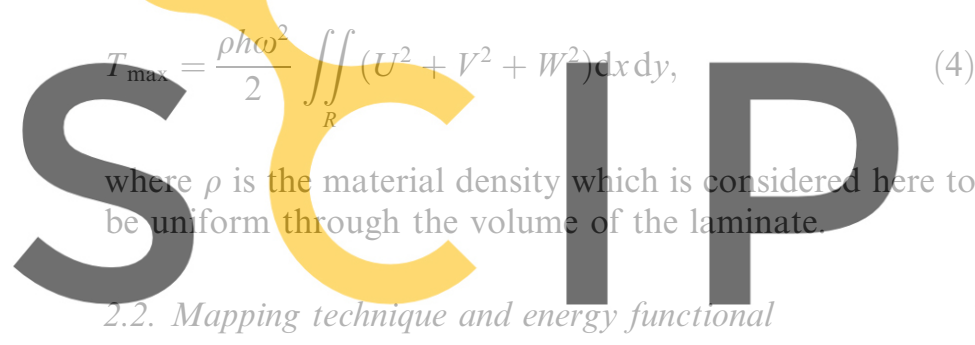

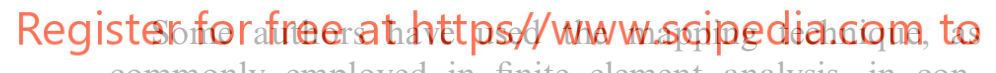
commonly employed in finite element analysis, in conjunction with other methods to study the dynamical behaviour of plates of various geometrical shapes, see for instance Ref. [14-19]. In all of these cases isotropic plates were considered. More recently, Nallim et al. [12] combined the mapping technique and the Ritz method to derive the eigenfrequency equation of symmetrically laminated plates. This methodology is extended and generalized here to be applied to unsymmetrically laminated plates.

The geometric mapping of a curvilinear quadrilateral region in the Cartesian $x-y$ plane (Fig. 1a) is accomplished from a square parent domain $-1 \leqslant \xi \leqslant 1$ and $-1 \leqslant \eta \leqslant 1$ in the natural $\xi-\eta$ plane (Fig. 1b), using the coordinate transformation:

$x=\sum_{i=1}^{n_{p}} N_{i}(\xi, \eta) x_{i}, \quad y=\sum_{i=1}^{n_{p}} N_{i}(\xi, \eta) y_{i}$

where $\left(x_{i}, y_{i}\right), i=1, \ldots, n_{p}$ are the coordinates of $n_{p}$ points on the boundary of the quadrilateral region $R$ and $N_{i}(\xi, \eta)$ are the interpolation functions [20].

Applying the chain rule of differentiation it can be shown that the first and the second derivatives of a function are related by
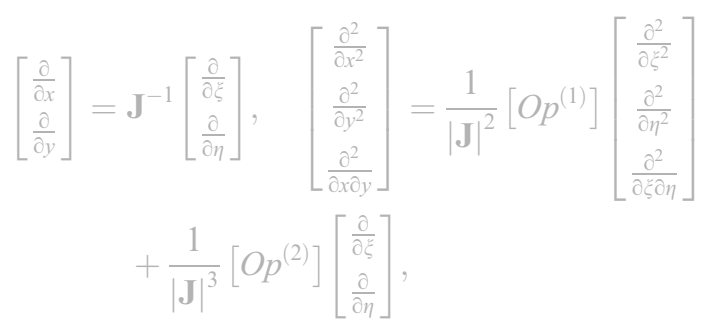

(6)

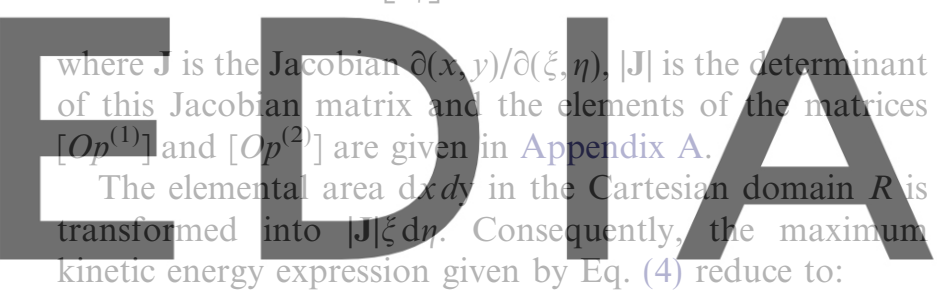

kinetic energy expression given by Eq. (4) reduce to:

downloadothel version without the watermark

where now $U=U(\xi, \eta), V=V(\xi, \eta)$ and $W=W(\xi, \eta)$.

Finally, substitution of the derivatives given by Eq. (6) into Eq. (3) leads to the following expressions for the maximum strain energy

$U_{\max }=U_{\max }^{(1)}+U_{\max }^{(2)}+U_{\max }^{(3)}$

where $U_{\max }^{(1)}$ contains the terms that correspond to the inplane effects, $U_{\max }^{(2)}$ those that correspond to the in-plane and out-of-plane coupling effects and $U_{\max }^{(3)}$ is the part of the strain energy that involves the bending and the twisting effects. Each one of these strain energies is given by the following expressions

$$
\begin{gathered}
U_{\max }^{(1)}=\frac{1}{2} \int_{-1}^{1} \int_{-1}^{1}\left(A_{1}^{*}\left(\frac{\partial U}{\partial \xi}\right)^{2}+A_{2}^{*}\left(\frac{\partial U}{\partial \eta}\right)^{2}+A_{3}^{*} \frac{\partial U}{\partial \xi} \frac{\partial U}{\partial \eta}\right. \\
+A_{4}^{*} \frac{\partial U}{\partial \xi} \frac{\partial V}{\partial \eta}+A_{5}^{*} \frac{\partial U}{\partial \xi} \frac{\partial V}{\partial \xi}+A_{6}^{*} \frac{\partial U}{\partial \eta} \frac{\partial V}{\partial \eta}+A_{7}^{*} \frac{\partial U}{\partial \eta} \frac{\partial V}{\partial \xi} \\
\left.+A_{8}^{*}\left(\frac{\partial V}{\partial \xi}\right)^{2}+A_{9}^{*}\left(\frac{\partial V}{\partial \eta}\right)^{2}+A_{10}^{*} \frac{\partial V}{\partial \xi} \frac{\partial V}{\partial \eta}\right)|\mathbf{J}|^{-1} \mathrm{~d} \xi \mathrm{d} \eta
\end{gathered}
$$




$$
\begin{aligned}
U_{\max }^{(2)}= & -\frac{1}{2} \int_{-1}^{1} \int_{-1}^{1}\left(\frac{\partial U}{\partial \xi} \frac{\partial^{2} W}{\partial \xi^{2}} B_{1}^{*}+\frac{\partial U}{\partial \xi} \frac{\partial^{2} W}{\partial \eta^{2}} B_{2}^{*}+\frac{\partial U}{\partial \eta} \frac{\partial^{2} W}{\partial \xi^{2}} B_{3}^{*}\right. \\
& +\frac{\partial U}{\partial \xi} \frac{\partial^{2} W}{\partial \xi \partial \eta} B_{4}^{*}+\frac{\partial U}{\partial \eta} \frac{\partial^{2} W}{\partial \eta^{2}} B_{5}^{*}+\frac{\partial U}{\partial \eta} \frac{\partial^{2} W}{\partial \xi \partial \eta} B_{6}^{*} \\
& +\frac{\partial V}{\partial \xi} \frac{\partial^{2} W}{\partial \xi^{2}} B_{11}^{*}+\frac{\partial V}{\partial \xi} \frac{\partial^{2} W}{\partial \eta^{2}} B_{12}^{*}+\frac{\partial V}{\partial \eta} \frac{\partial^{2} W}{\partial \xi^{2}} B_{13}^{*} \\
& \left.+\frac{\partial V}{\partial \xi} \frac{\partial^{2} W}{\partial \xi \partial \eta} B_{14}^{*}+\frac{\partial V}{\partial \eta} \frac{\partial^{2} W}{\partial \eta^{2}} B_{15}^{*}+\frac{\partial V}{\partial \eta} \frac{\partial^{2} W}{\partial \xi \partial \eta} B_{16}^{*}\right) \\
& \times|\mathbf{J}|^{-2} \mathrm{~d} \xi \mathrm{d} \eta-\frac{1}{2} \int_{-1}^{1} \int_{-1}^{1}\left(\frac{\partial U}{\partial \xi} \frac{\partial W}{\partial \xi} B_{7}^{*}+\frac{\partial U}{\partial \xi} \frac{\partial W}{\partial \eta} B_{8}^{*}\right. \\
& +\frac{\partial U}{\partial \eta} \frac{\partial W}{\partial \xi} B_{9}^{*}+\frac{\partial U}{\partial \eta} \frac{\partial W}{\partial \eta} B_{10}^{*}+\frac{\partial V}{\partial \xi} \frac{\partial W}{\partial \xi} B_{17}^{*}+\frac{\partial V}{\partial \xi} \frac{\partial W}{\partial \eta} B_{18}^{*} \\
& \left.+\frac{\partial V}{\partial \eta} \frac{\partial W}{\partial \xi} B_{19}^{*}+\frac{\partial V}{\partial \eta} \frac{\partial W}{\partial \eta} B_{20}^{*}\right)|\mathbf{J}|^{-3} \mathrm{~d} \xi \mathrm{d} \eta
\end{aligned}
$$
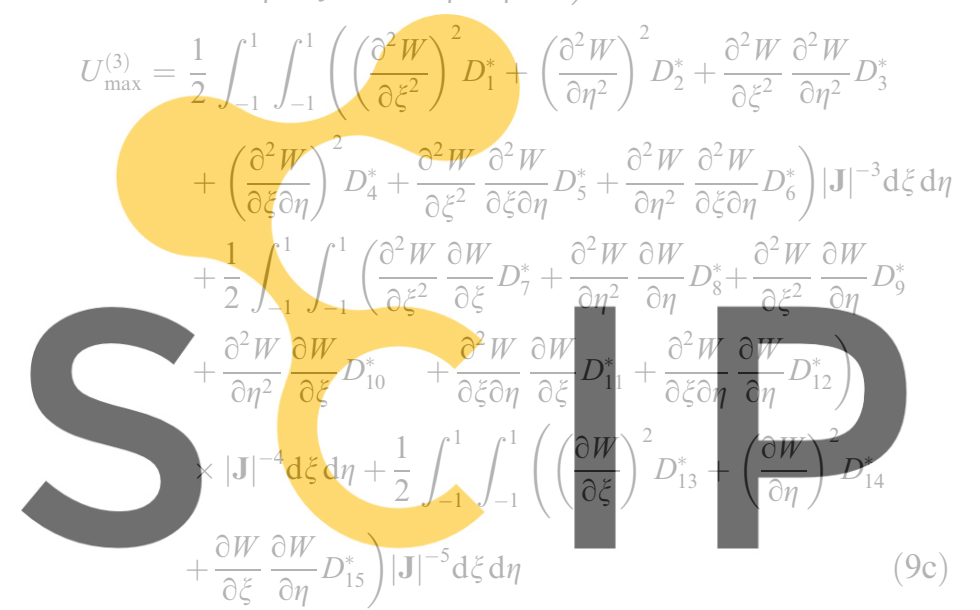

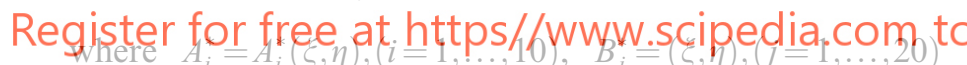
and $D_{k}^{*}=(\xi, \eta),(k=1, \ldots, 15)$ are functions that depend on the problem parameters, i.e., plate geometry and material properties, and are defined in Appendix B.

The energy functional for free vibration of the plate is given by:

$F=U_{\max }-T_{\max }$,

which is to be minimized according to the Ritz principle, as will be discussed in Section 3.

\subsection{Boundary conditions and approximating functions}

The situation for unsymmetrically laminated plates is more complex than for symmetric ones because the transverse and in-plane vibrations are coupled. Actually, there are four types of boundary conditions that can be called simply supported (S), clamped (C) or free edges (F) [21]. The various combinations of these constraints are summarized in Table 1.

In the application of the Ritz method only the essential boundary conditions are required to be satisfied by the assumed functions [22]. The fact that the natural boundary conditions need not be satisfied by the chosen coordinate functions is a very important characteristic of the Ritz method, specially when dealing with problems for which these satisfaction is very difficult to achieve $[23,24]$.

The use of beam orthogonal polynomials to study anisotropic plates is very satisfactory, as has been demonstrated through some works $[12,25,26]$ since the procedure has a quick convergence to the solution and takes place practically without oscillations. For this reason, in the present paper the three field displacement components are expressed in terms of the natural coordinates system by sets of beam characteristic orthogonal polynomials, $\left\{p_{i}^{(u)}(\xi)\right\}$, $\left\{q_{j}^{(u)}(\eta)\right\},\left\{p_{i}^{(v)}(\xi)\right\},\left\{q_{j}^{(v)}(\eta)\right\},\left\{p_{i}^{(w)}(\xi)\right\}$ and $\left\{q_{j}^{(w)}(\eta)\right\}$, as

$$
\begin{aligned}
& U(\xi, \eta) \approx U_{M N}(\xi, \eta)=\sum_{i=1}^{M} \sum_{j=1}^{N} c_{i j}^{(u)} p_{i}^{(u)}(\xi) q_{j}^{(u)}(\eta), \\
& V(\xi, \eta) \approx V_{M N}(\xi, \eta)=\sum_{i=1}^{M} \sum_{j=1}^{N} c_{i j}^{(v)} p_{i}^{(v)}(\xi) q_{j}^{(v)}(\eta), \\
& W(\xi, \eta) \approx W_{M N}(\xi, \eta)=\sum_{i=1}^{M} \sum_{j=1}^{N} c_{i j}^{(w)} p_{i}^{(w)}(\xi) q_{j}^{(w)}(\eta),
\end{aligned}
$$

where $c_{i j}^{(u)}, c_{i j}^{(v)}$ and $c_{i j}^{(w)}$ are the unknown coefficients, and $M$ and $N$ are the numbers of polynomials in each natural

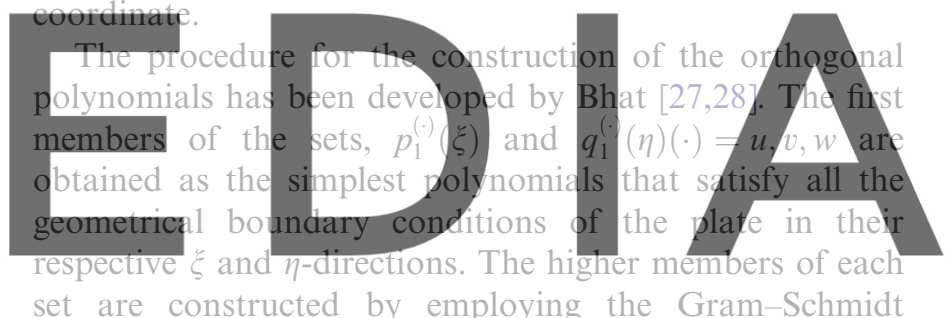

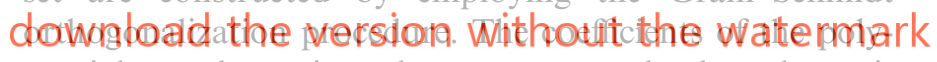
nomials are chosen in such a way as to make the polynomials orthonormal.

It is important to point out that working with the master element in natural coordinates allows us to use the same sets of orthogonal polynomials for plates of different shapes. This fact makes possible a unified treatment.

\section{Application of the Ritz method for the study of free vibration}

The Ritz method is applied to determine analytical approximate solutions for general laminated plates of different shapes. For the dynamical analysis the Ritz procedure requires the minimization of the energy functional (Eq. (10)) with respect to each of the $c_{i j}^{(u)}, c_{i j}^{(v)}$ and $c_{i j}^{(w)}$ coefficients

$\frac{\partial F}{\partial c_{i j}^{(u)}}=0, \quad \frac{\partial F}{\partial c_{i j}^{(v)}}=0, \quad \frac{\partial F}{\partial c_{i j}^{(w)}}=0, \quad(i, j=1, \ldots, M, N)$

Substituting Eqs. (11a)-(11c) into the expressions for $U_{\max }$ (Eq. (8)) and $T_{\max }$ (Eq. (7)), and subsequently applying Eq. (12) result in the following governing eigenvalue equation: 
Table 1

Notations for various combinations of boundary conditions, in which $n$ and $s$ indicate the directions normal and tangential to the respective plate edges Transverse boundary supports

\section{In-plane constraints}

\begin{tabular}{llll}
\hline$u_{n}=0, u_{s}=0$ & $N_{n}=0, u_{s}=0$ & $u_{n}=0, N_{n s}=0$ & $N_{n}=0, N_{n s}=0$ \\
$\mathrm{C}_{1}$ & $\mathrm{C}_{2}$ & $\mathrm{C}_{3}$ & $\mathrm{C}_{4}$ \\
$\mathrm{~S}_{1}$ & $\mathrm{~S}_{2}$ & $\mathrm{~S}_{3}$ & $\mathrm{~S}_{4}$ \\
$\mathrm{~F}_{1}$ & $\mathrm{~F}_{2}$ & $\mathrm{~F}_{3}$ & $\mathrm{~F}_{4}$ \\
\hline
\end{tabular}

$\left(\mathbf{K}-\omega^{2} \mathbf{M}\right)\{\mathbf{C}\}=\{\mathbf{0}\}$.

In Eq. (13), $\mathbf{K}$ is the stiffness matrix, $\mathbf{M}$ is the mass matrix and $\{\mathbf{C}\}$ is the unknown coefficient vector, and are respectively given by

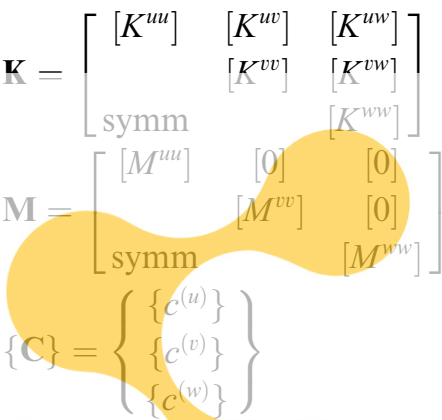

$(14 a)$

$$
\begin{aligned}
K_{i j m n}^{w w}= & \int_{-1}^{1} \int_{-1}^{1}\left(2 D_{1}^{*} P_{i m}^{w w 22} Q_{j n}^{w w 00}+2 D_{2}^{*} P_{i m}^{w w 00} Q_{j n}^{w w 22}\right. \\
& +D_{3}^{*}\left(P_{i m}^{w w 02} Q_{j n}^{w w 20}+P_{i m}^{w w 20} Q_{j n}^{w w 02}\right)+2 D_{4}^{*} P_{i m}^{w w 11} Q_{j n}^{w w 11} \\
& +D_{5}^{*}\left(P_{i m}^{w w 12} Q_{j n}^{w w 10}+P_{i m}^{w w 21} Q_{j n}^{w w 01}\right)+D_{6}^{*}\left(P_{i m}^{w w 10} Q_{j n}^{w w 12}\right.
\end{aligned}
$$
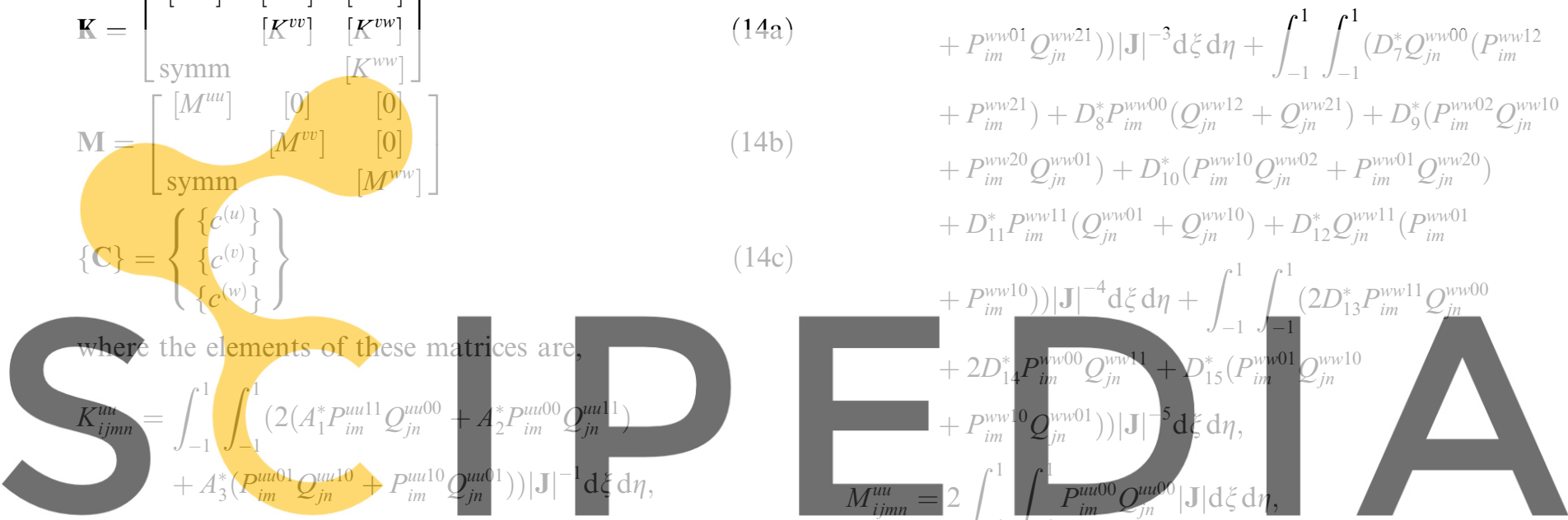

$\left.+P_{i m}^{w w 20} Q_{j n}^{w w 01}\right)+D_{10}^{*}\left(P_{i m}^{w w 10} Q_{j n}^{w w 02}+P_{i m}^{w w 01} Q_{j n}^{w w 20}\right)$

$+D_{11}^{*} P_{i m}^{w w 11}\left(Q_{j n}^{w w 01}+Q_{j n}^{w w 10}\right)+D_{12}^{*} Q_{j n}^{w w 11}\left(P_{i m}^{w w 01}\right.$

$K_{i j m n}^{u v}=\int^{1} \int^{1}\left(A_{4}^{*} P_{i m 1}^{u v 10} Q_{j n}^{u v 01}+A_{5}^{*} P_{i m}^{u v 11} Q_{j n}^{u v 00}+A_{6}^{*} P_{i m}^{u v 00} Q_{j n}^{u v 1}\right.$

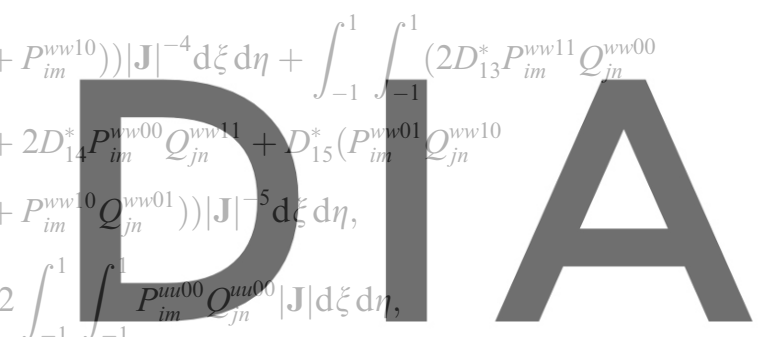

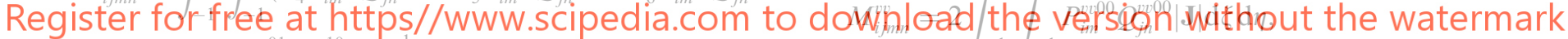

$\left.+A_{7}^{*} P_{i m}^{u v 01} Q_{j n}^{u v 10}\right)|\boldsymbol{J}|^{-1} \mathrm{~d} \xi \mathrm{d} \eta$,

$$
\begin{aligned}
K_{i j m n}^{u w}= & -\int_{-1}^{1} \int_{-1}^{1}\left(B_{1}^{*} P_{i m}^{u w 12} Q_{j n}^{u w 00}+B_{2}^{*} P_{i m}^{u w 10} Q_{j n}^{u w 02}\right. \\
& +B_{3}^{*} P_{i m}^{u w 02} Q_{j n}^{u w 10}+B_{4}^{*} P_{i m}^{u w 11} Q_{j n}^{u w 01}+B_{5}^{*} P_{i m}^{u w 00} Q_{j n}^{u w 12} \\
& \left.+B_{6}^{*} P_{i m}^{u w 01} Q_{j n}^{u w 11}\right)|\mathbf{J}|^{-2} \mathrm{~d} \xi \mathrm{d} \eta-\int_{-1}^{1} \int_{-1}^{1}\left(B_{7}^{*} P_{i m}^{u w 11} Q_{j n}^{u w 00}\right. \\
& +B_{8}^{*} P_{i m}^{u w 10} Q_{j n}^{u w 01}+B_{9}^{*} P_{i m}^{u w 01} Q_{j n}^{u w 10} \\
& \left.+B_{10}^{*} P_{i m}^{u w 00} Q_{j n}^{u w 11}\right)|\mathbf{J}|^{-3} \mathrm{~d} \xi \mathrm{d} \eta \\
K_{i j m n}^{v v} & \int_{-1}^{1} \int_{-1}^{1}\left(2\left(A_{8}^{*} P_{i m}^{v v 11} Q_{j n}^{v v 00}+A_{9}^{*} P_{i m}^{v v 00} Q_{j n}^{v v 11}\right)\right. \\
& \left.+A_{10}^{*}\left(P_{i m}^{v v 10} Q_{j n}^{v v 01}+P_{i m}^{v v 01} Q_{j n}^{v v 10}\right)\right)|\mathbf{J}|^{-1} \mathrm{~d} \xi \mathrm{d} \eta \\
K_{i j m n}^{v w}= & -\int_{-1}^{1} \int_{-1}^{1}\left(B_{11}^{*} P_{i m}^{v w 12} Q_{j n}^{v w 00}+B_{12}^{*} P_{i m}^{v w 10} Q_{j n}^{v w 02}\right. \\
& +B_{13}^{*} P_{i m}^{v w 02} Q_{j n}^{v w 10}+B_{14}^{*} P_{i m}^{v w 11} Q_{j n}^{v w 01}+B_{15}^{*} P_{i m}^{v w 00} Q_{j n}^{v w 12} \\
& \left.+B_{16}^{*} P_{i m}^{v w 01} Q_{j n}^{v w 11}\right)|\mathbf{J}|^{-2} \mathrm{~d} \xi \mathrm{d} \eta-\int_{-1}^{1} \int_{-1}^{1}\left(B_{17}^{*} P_{i m}^{v w 11} Q_{j n}^{v w 00}\right. \\
& +B_{18}^{*} P_{i m}^{v w 10} Q_{j n}^{v w 01}+B_{19}^{*} P_{i m}^{v w 01} Q_{j n}^{v w 10} \\
& \left.+B_{20}^{*} P_{i m}^{v w 00} Q_{j n}^{v w 11}\right)|\mathbf{J}|^{-3} \mathrm{~d} \xi \mathrm{d} \eta,
\end{aligned}
$$

$M_{i j m n}^{w w}=2 \int_{-1}^{1} \int_{-1}^{1} P_{i m}^{w w 00} Q_{j n}^{w w 00}|\boldsymbol{J}| \mathrm{d} \xi \mathrm{d} \eta$

$i, m=1, \ldots, M ; \quad j, n=1, \ldots, N$,

in which

$$
\begin{aligned}
& P_{\text {im }}^{u u r s}=\frac{\mathrm{d}^{r} p_{i}^{(u)}}{\mathrm{d} \xi^{r}} \frac{\mathrm{d}^{s} p_{m}^{(u)}}{\mathrm{d} \xi^{s}}, \quad Q_{j n}^{u u r s}=\frac{\mathrm{d}^{r} q_{j}^{(u)}}{\mathrm{d} \eta^{r}} \frac{\mathrm{d}^{s} q_{n}^{(u)}}{\mathrm{d} \eta^{s}}, \\
& P_{i m}^{u v r s}=\frac{\mathrm{d}^{r} p_{i}^{(u)}}{\mathrm{d} \xi^{r}} \frac{\mathrm{d}^{s} p_{m}^{(v)}}{\mathrm{d} \xi^{s}}, \quad Q_{j n}^{u v r s}=\frac{\mathrm{d}^{r} q_{j}^{(u)}}{\mathrm{d} \eta^{r}} \frac{\mathrm{d}^{s} q_{n}^{(v)}}{\mathrm{d} \eta^{s}} \\
& P_{i m}^{u w r s}=\frac{\mathrm{d}^{r} p_{i}^{(u)}}{\mathrm{d} \xi^{r}} \frac{\mathrm{d}^{s} p_{m}^{(w)}}{\mathrm{d} \xi^{s}}, \quad Q_{j n}^{u w r s}=\frac{\mathrm{d}^{r} q_{j}^{(u)}}{\mathrm{d} \eta^{r}} \frac{\mathrm{d}^{s} q_{n}^{(w)}}{\mathrm{d} \eta^{s}}, \\
& P_{i m}^{v v r s}=\frac{\mathrm{d}^{r} p_{i}^{(v)}}{\mathrm{d} \xi^{r}} \frac{\mathrm{d}^{s} p_{m}^{(v)}}{\mathrm{d} \xi^{s}}, \quad Q_{j n}^{v v r s}=\frac{\mathrm{d}^{r} q_{j}^{(v)}}{\mathrm{d} \eta^{r}} \frac{\mathrm{d}^{s} q_{n}^{(v)}}{\mathrm{d} \eta^{s}}, \\
& P_{\text {im }}^{v w r s}=\frac{\mathrm{d}^{r} p_{i}^{(v)}}{\mathrm{d} \xi^{r}} \frac{\mathrm{d}^{s} p_{m}^{(w)}}{\mathrm{d} \xi^{s}}, \quad Q_{j n}^{v w r s}=\frac{\mathrm{d}^{r} q_{j}^{(v)}}{\mathrm{d} \eta^{r}} \frac{\mathrm{d}^{s} q_{n}^{(w)}}{\mathrm{d} \eta^{s}}, \\
& P_{i m}^{w w r s}=\frac{\mathrm{d}^{r} p_{i}^{(w)}}{\mathrm{d} \xi^{r}} \frac{\mathrm{d}^{s} p_{m}^{(w)}}{\mathrm{d} \xi^{s}}, \quad Q_{j n}^{w w r s}=\frac{\mathrm{d}^{r} q_{j}^{(w)}}{\mathrm{d} \eta^{r}} \frac{\mathrm{d}^{s} q_{n}^{(w)}}{\mathrm{d} \eta^{s}}, \\
& r, s=0,1,2
\end{aligned}
$$


a

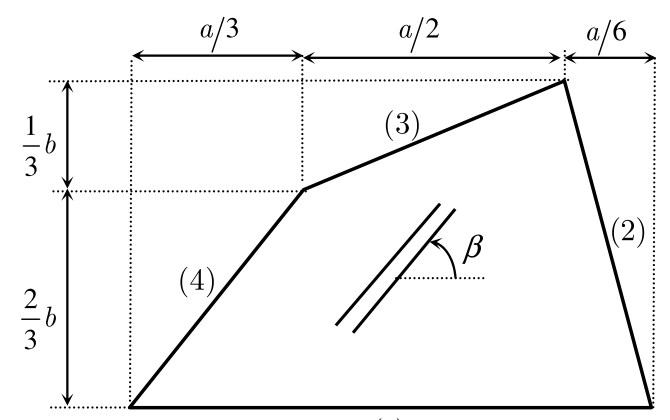

(1)

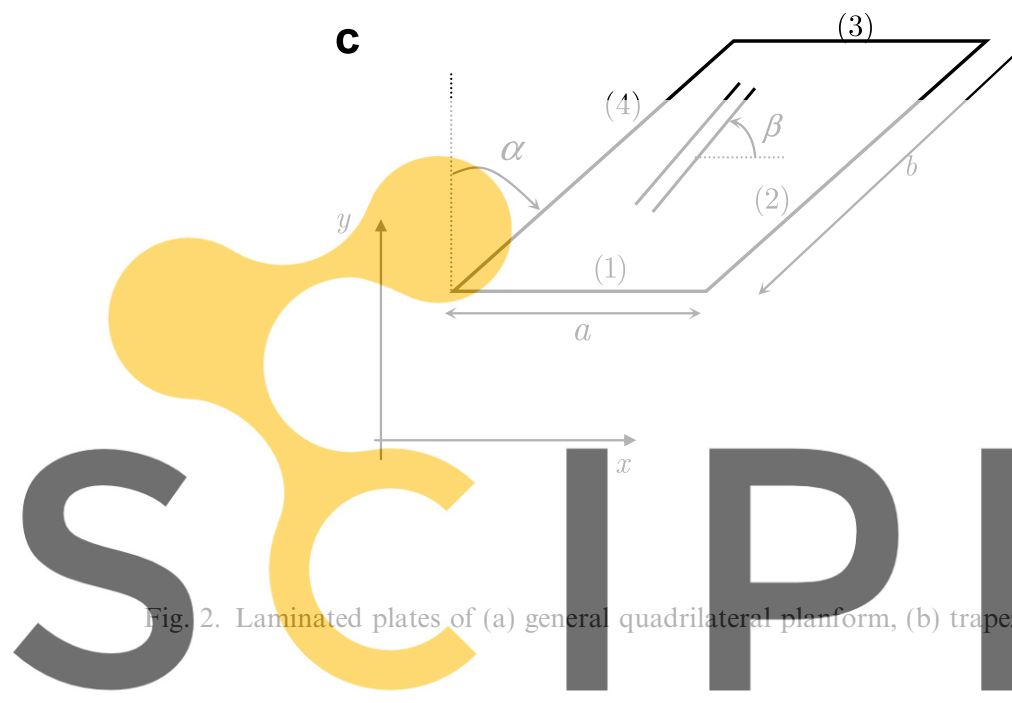

Eq. (13) yields an eigenvalue determinant, whose zeros

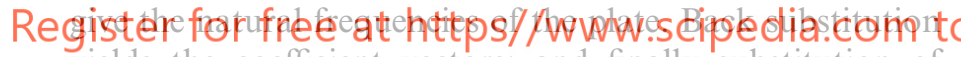
yields the coefficient vectors; and finally substitution of these coefficient vectors into Eq. (11) gives the corresponding free vibration mode shapes.

\section{Verification of the formulation and its numerical applications}

\subsection{General description}

The variational algorithm developed in this paper was programmed and used for the free vibration analysis of unsymmetrically laminated thin plates having different shapes, geometric parameters, stacking sequences, material properties and boundary conditions. The examples considered in this study are confined to laminates with layers of equal thickness, even though the procedure was formulated for plies with arbitrary thickness.

Let us introduce the terminology to be used throughout the remainder of the paper for describing the boundary conditions of the considered plates. The designation $\mathrm{C}_{i} \mathrm{~S}_{i-}$ $\mathrm{F}_{i} \mathrm{~S}_{i}$, for example, identifies a plate with edges (1) clamped, (2) simply supported, (3) free and (4) simply supported (see Fig. 2), the subscript $i(i=1, \ldots, 4)$ identifies the in-plane constraints according to Table 1 . The reference flexural stiffness is $D_{\beta}=E_{L} h^{3} / 12\left(1-v_{L T} v_{T L}\right)$ and the results are b

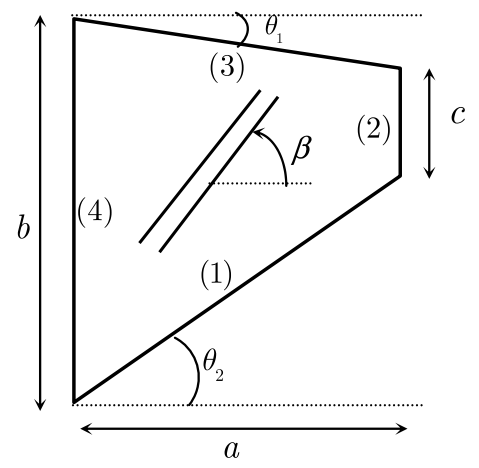

d

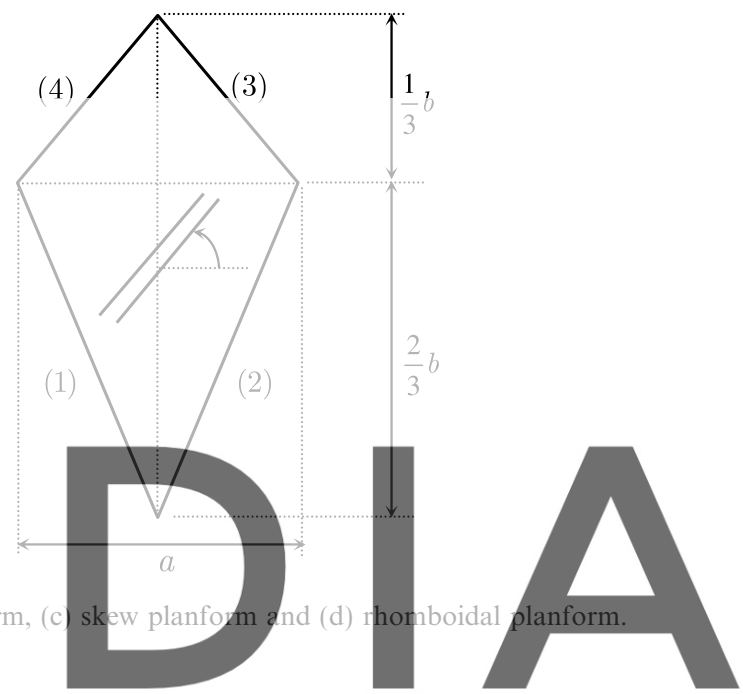

presented for a representative high modulus graphite/

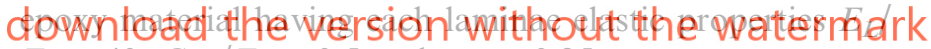
$E_{T}=40 ; G_{L T} / E_{T}=0.5$ and $v_{L T}=0.25$.

The main purposes of the numerical applications presented in this section are twofold. One is to demonstrate the accuracy, the flexibility and the efficiency of the proposed method and the other is to produce some results which may be regarded as benchmark solutions for other academic research workers and design engineers.

\subsection{Validation and convergence studies}

In order to evaluate the accuracy and reliability of the present method, comparison and convergence studies are carried out in this section. Convergence studies have been undertaken for simply supported $\left(\mathrm{S}_{2} \mathrm{~S}_{2} \mathrm{~S}_{2} \mathrm{~S}_{2}\right)$, fully clamped $\left(\mathrm{C}_{1} \mathrm{C}_{1} \mathrm{C}_{1} \mathrm{C}_{1}\right)$, cantilever $\left(\mathrm{F}_{4} \mathrm{~F}_{4} \mathrm{~F}_{4} \mathrm{C}_{1}\right)$ and for $\mathrm{C}_{3} \mathrm{~S}_{3} \mathrm{C}_{3} \mathrm{~S}_{3}$ general quadrilateral laminated plates with $a / b=2$ (Fig. 2a). Two layer laminates (in this case in-plane stretching effects are more important) with a width-thickness $a / h=1000$ are considered. Results for antisymmetric angle-ply $\left(30^{\circ} /-30^{\circ}\right)$ laminated plates are given in Table 2. The number of polynomials in each natural coordinate for $u, v$ and $w$ are stepped steadily from 6 to 12 to demonstrate the downward convergence of the first eight no dimensional frequency parameters $\Omega_{i}=\omega_{i} a^{2} \sqrt{\rho h / D_{\beta}}$. It can be seen that the eigenfrequencies converge monotonically from above as 
Table 2

Convergence of frequency parameters $\Omega=\omega a^{2} \sqrt{\rho h / D_{\beta}}$ for general quadrilateral angle-ply $\left(30^{\circ} /-30^{\circ}\right)$ graphite/epoxy plates $(a / b=2, h / a=0.001)$

\begin{tabular}{|c|c|c|c|c|c|c|c|c|}
\hline$M \times N$ & $\Omega_{1}$ & $\Omega_{2}$ & $\Omega_{3}$ & $\Omega_{4}$ & $\Omega_{5}$ & $\Omega_{6}$ & $\Omega_{7}$ & $\Omega_{8}$ \\
\hline \multicolumn{9}{|l|}{$\mathrm{S}_{2} \mathrm{~S}_{2} \mathrm{~S}_{2} \mathrm{~S}_{2}$} \\
\hline $6 \times 6$ & 31.327 & 54.395 & 70.445 & 97.240 & 115.548 & 137.559 & 147.174 & 181.248 \\
\hline $7 \times 7$ & 31.266 & 54.255 & 70.006 & 94.603 & 114.142 & 135.206 & 142.983 & 169.465 \\
\hline $8 \times 8$ & 31.213 & 54.171 & 69.842 & 94.330 & 113.360 & 134.373 & 139.487 & 165.261 \\
\hline $9 \times 9$ & 31.173 & 54.134 & 69.715 & 94.147 & 113.219 & 134.611 & 138.865 & 163.449 \\
\hline $10 \times 10$ & 31.140 & 54.104 & 69.613 & 94.128 & 113.088 & 134.537 & 138.519 & 163.183 \\
\hline $11 \times 11$ & 31.112 & 54.080 & 69.528 & 94.117 & 113.014 & 134.505 & 138.500 & 162.930 \\
\hline $12 \times 12$ & 31.089 & 54.061 & 69.457 & 94.110 & 112.954 & 134.482 & 138.485 & 162.906 \\
\hline \multicolumn{9}{|c|}{$\mathrm{C}_{1} \mathrm{C}_{1} \mathrm{C}_{1} \mathrm{C}_{1}$} \\
\hline $6 \times 6$ & 45.505 & 79.021 & 97.883 & 124.339 & 146.878 & 171.814 & 184.440 & 217.912 \\
\hline $7 \times 7$ & 45.392 & 79.490 & 97.121 & 123.365 & 145.582 & 171.265 & 178.549 & 209.197 \\
\hline $8 \times 8$ & 45.344 & 79.397 & 97.951 & 123.252 & 145.948 & 170.587 & 177.495 & 206.356 \\
\hline $9 \times 9$ & 45.320 & 79.369 & 97.880 & 123.945 & 145.182 & 170.082 & 176.846 & 205.641 \\
\hline $10 \times 10$ & 45.305 & 79.353 & 97.844 & 123.911 & 145.995 & 170.953 & 176.044 & 204.852 \\
\hline $11 \times 11$ & 45.297 & 79.344 & 97.826 & 123.903 & 145.924 & 170.910 & 176.849 & 204.236 \\
\hline $12 \times 12$ & 45.292 & 79.338 & 97.815 & 123.901 & 145.886 & 170.892 & 176.822 & 204.076 \\
\hline \multicolumn{9}{|c|}{$\mathrm{F}_{4} \mathrm{~F}_{4} \mathrm{~F}_{4} \mathrm{C}_{1}$} \\
\hline $6 \times 6$ & 2.8963 & 9.1009 & 15.993 & 27.095 & 36.035 & 45.279 & 60.738 & 70.460 \\
\hline $7 \times 7$ & 2.8857 & 8.9957 & 15.912 & 26.816 & 35.721 & 44.852 & 60.172 & 68.407 \\
\hline $8 \times 8$ & 2.8770 & 8.9385 & 15.866 & 26.774 & 35.610 & 44.691 & 59.062 & 67.620 \\
\hline $9 \times 9$ & 2.8709 & 8.9030 & 15.841 & 26.748 & 35.549 & 44.604 & 59.018 & 67.533 \\
\hline $10 \times 10$ & 2.8661 & 8.8792 & 15.825 & 26.730 & 35.510 & 44.555 & 58.982 & 67.502 \\
\hline $11 \times 11$ & 2.8623 & 8.8631 & 15.815 & 26.717 & 35.484 & 44.522 & 58.965 & 67.482 \\
\hline & 2.859 & 8.8517 & & & 35.466 & & 58.953 & \\
\hline 6 & 38.701 & 64.46 & 91.873 & 104.481 & 134.1 & & 168.978 & \\
\hline & 38.627 & 64.252 & 91.006 & 102.484 & 130.632 & & 166.459 & \\
\hline & 38.577 & 64.150 & 90.961 & 101.703 & 129.835 & & 163.003 & \\
\hline 9 & 38.541 & 64.089 & 90.924 & 101.592 & 129.367 & 80.424 & 162.87 & 78 \\
\hline $11 \times 11$ & 38.496 & 64.014 & 90.896 & 101.562 & 129.128 & 150.315 & 162.709 & 178.067 \\
\hline $12 \times 12$ & 38.481 & 63.990 & 90.887 & 101.558 & 129.056 & 150.299 & 162.703 & 177.940 \\
\hline
\end{tabular}

the increase of the number of terms of the trial functions. In general, 10-12 terms of the trial functions can give sufficiently satisfactory results for first eight eigenfrequencies.

The accuracy and reliability of the results obtained with the present approach are next demonstrated by comparing them with some selected values published by other researchers for unsymmetrically laminated trapezoidal and skew plates.

The comparison presented in Table 3, authenticates the validity of the present method for antisymmetric laminated plates with symmetric trapezoidal planform $\theta_{1}=\theta_{2}$ (see Fig. 2b). The first eight non-dimensional frequencies $\omega a^{2} \sqrt{\rho h / D_{\beta}}$ for $\mathrm{F}_{4} \mathrm{~F}_{4} \mathrm{~F}_{4} \mathrm{C}_{1}$ two-ply $\left(30^{\circ} /-30^{\circ}\right)$ and fourply $\left(30^{\circ} /-30^{\circ} / 30^{\circ} \%-30^{\circ}\right)$ antisymmetric laminated graphite-epoxy plates are compared with those of Liew and Lim [8], and very good agreement is obtained.

The second example verifies the accuracy of the eigenvalues for thin skew fibre reinforced antisymmetric angleply $\left(45^{\circ} /-45^{\circ} / 45^{\circ} /-45^{\circ}\right)$ plates and cross-ply $\left(90^{\circ} / 0^{\circ} / 90^{\circ} /\right.$ $0^{\circ}$ ) plates. The plate geometry is defined by means of $a, b$ and $\alpha$ as shown in Fig. 2c. The material properties of each lamina correspond to graphite/epoxy and two skew angles, i.e., $\alpha=30^{\circ}, 45^{\circ}$ are used for the comparisons. The first eight non-dimensional frequencies $\hat{\Omega}_{i}=\left(\omega_{i} a^{2} / h \pi^{2}\right) \sqrt{\rho / E_{T}}$ obtained with the present approach for fully clamped $\left(\mathrm{C}_{1} \mathrm{C}_{1} \mathrm{C}_{1} \mathrm{C}_{1}\right)$, are compared with the solutions of Wang [10] in Table 4, and excellent agreement is achieved between both solutions.

From the convergence analysis and the comparisons performed it is clear that $M, N=10$ produces no drastic change in the solutions compared with $M, N=11-12$. Therefore, in the next section it was decided to use $M$, $N=10$ to generate results with sufficient accuracy from an engineering viewpoint.

\subsection{Numerical results and discussion}

The developed Ritz formulation is applied in this section to obtain the natural frequencies of free vibration and modal shapes of general rhomboidal laminated plates as shown in Fig. 2d. The planform geometries of these plates are defined by means of the aspect ratio $b / a$, while boundaries having different combinations of in-plane and/or transverse constraints are analyzed. The non-dimensional frequency parameter is given by $\Omega_{i}=\omega_{i} a^{2} \sqrt{\rho h / D_{\beta}}$, and results are presented for the representative high modulus 
Table 3

Frequency parameters $\Omega=\omega a^{2} \sqrt{\rho h / D_{\beta}}$ for cantilever $\left(\mathrm{F}_{4} \mathrm{~F}_{4} \mathrm{~F}_{4} \mathrm{C}_{1}\right)$ trapezoidal laminated graphite/epoxy plates $\left(a / b=1, \theta_{1}=\theta_{2}, h / a=0.001\right)$

\begin{tabular}{|c|c|c|c|c|c|c|c|c|c|}
\hline$c / b$ & Sources & $\Omega_{1}$ & $\Omega_{2}$ & $\Omega_{3}$ & $\Omega_{4}$ & $\Omega_{5}$ & $\Omega_{6}$ & $\Omega_{7}$ & $\Omega_{8}$ \\
\hline \multicolumn{10}{|c|}{$\left(30^{\circ} /-30^{\circ}\right)$} \\
\hline \multirow[t]{3}{*}{0.25} & Present $(10 \times 10)$ & 2.0616 & 8.5508 & 9.7945 & 22.189 & 23.319 & 25.712 & 40.875 & 44.525 \\
\hline & Present $(11 \times 11)$ & 2.0616 & 8.5507 & 9.7943 & 22.188 & 23.318 & 25.711 & 40.872 & 44.524 \\
\hline & Ref. [8] & 2.0616 & 8.5506 & 9.7942 & 22.187 & 23.318 & 25.710 & 40.870 & 44.523 \\
\hline \multirow[t]{3}{*}{0.50} & Present $(10 \times 10)$ & 1.7646 & 6.1301 & 9.2878 & 16.288 & 17.378 & 25.533 & 32.120 & 33.098 \\
\hline & Present $(11 \times 11)$ & 1.7646 & 6.1298 & 9.2873 & 16.288 & 17.377 & 25.529 & 32.118 & 33.098 \\
\hline & Ref. [8] & 1.7645 & 6.1297 & 9.2871 & 16.288 & 17.376 & 25.528 & 32.118 & 33.097 \\
\hline \multirow[t]{3}{*}{0.75} & Present $(10 \times 10)$ & 1.5955 & 4.6155 & 8.8041 & 11.516 & 14.701 & 22.791 & 24.550 & 26.828 \\
\hline & Present $(11 \times 11)$ & 1.5954 & 4.6151 & 8.8018 & 11.515 & 14.700 & 22.791 & 24.539 & 26.826 \\
\hline & Ref. [8] & 1.5954 & 4.6153 & 8.8028 & 11.516 & 14.701 & 22.791 & 24.543 & 26.827 \\
\hline \multirow[t]{3}{*}{1.0} & Present $(10 \times 10)$ & 1.4803 & 3.6343 & 7.4610 & 9.5804 & 12.898 & 15.907 & 21.513 & 25.532 \\
\hline & Present $(11 \times 11)$ & 1.4800 & 3.6339 & 7.4574 & 9.5792 & 12.897 & 15.907 & 21.507 & 25.520 \\
\hline & Ref. [8] & 1.4803 & 3.6351 & 7.4611 & 9.5805 & 12.901 & 15.907 & 21.513 & 25.524 \\
\hline \multicolumn{10}{|c|}{$\left(30^{\circ} /-30^{\circ} / 30^{\circ} /-30^{\circ}\right)$} \\
\hline \multirow[t]{3}{*}{0.25} & Present $(10 \times 10)$ & 3.2045 & 13.123 & 14.897 & 33.815 & 33.821 & 39.900 & 62.762 & 64.676 \\
\hline & Present $(11 \times 11)$ & 3.2044 & 13.123 & 14.896 & 33.815 & 33.819 & 39.897 & 62.757 & 64.676 \\
\hline & Ref. [8] & 3.2044 & 13.123 & 14.896 & 33.815 & 33.819 & 39.896 & 62.754 & 64.673 \\
\hline \multirow[t]{3}{*}{0.50} & Present $(10 \times 10)$ & 2.7513 & 9.4506 & 14.142 & 23.701 & 26.434 & 39.829 & 46.550 & 48.559 \\
\hline & Present $(11 \times 11)$ & 2.7512 & 9.4503 & 14.141 & 23.701 & 26.432 & 39.816 & 46.550 & 48.551 \\
\hline & Ref. [8] & 2.7512 & 9.4502 & 14.141 & 23.701 & 26.432 & 39.815 & 46.549 & 48.551 \\
\hline \multirow[t]{5}{*}{0.75} & Present $(10 \times 10)$ & 2.4896 & 7.1546 & 13.200 & 17.319 & 22.197 & 31.929 & 37.994 & 41.241 \\
\hline & $\begin{array}{l}\text { Present }(11 \times 11) \\
\text { Ref. }[8]\end{array}$ & $\begin{array}{l}2.4895 \\
2.4895\end{array}$ & & & $\begin{array}{l}17.318 \\
17.310\end{array}$ & 22.195 & 31.929 & $\begin{array}{l}37.962 \\
37.971\end{array}$ & \\
\hline & Present $(10 \times 10)$ & 2.3083 & 6722 & 0.961 & 14.882 & 8.916 & .060 & 33.168 & \\
\hline & Present $(11 \times 11)$ & 2.3077 & 5.6716 & 10.950 & 14.87 & 8.914 & .059 & 33.15 & \\
\hline & Ref. [8] & 2.3083 & 5.6740 & 10.959 & 14.881 & 8.922 & 3.060 & 3216 & \\
\hline
\end{tabular}

Register for free at https//www.scipedia.com to download the version without the watermark

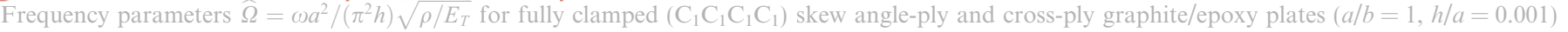

\begin{tabular}{|c|c|c|c|c|c|c|c|c|c|}
\hline$\alpha$ & Sources & $\widehat{\Omega}_{1}$ & $\widehat{\Omega}_{2}$ & $\widehat{\Omega}_{3}$ & $\widehat{\Omega}_{4}$ & $\widehat{\Omega}_{5}$ & $\widehat{\Omega}_{6}$ & $\widehat{\Omega}_{7}$ & $\widehat{\Omega}_{8}$ \\
\hline \multicolumn{10}{|c|}{$\left(45^{\circ} /-45^{\circ} / 45^{\circ} /-45^{\circ}\right)$} \\
\hline \multirow[t]{3}{*}{$30^{\circ}$} & Present $(10 \times 10)$ & 4.8793 & 8.3836 & 11.123 & 12.563 & 16.341 & 17.469 & 20.082 & 22.503 \\
\hline & Present $(11 \times 11)$ & 4.8793 & 8.3836 & 11.123 & 12.563 & 16.341 & 17.469 & 20.081 & 22.502 \\
\hline & Ref. $[10]$ & 4.8889 & 8.4053 & 11.1461 & 12.5901 & 16.3995 & 17.5057 & 20.1206 & 22.5803 \\
\hline \multirow[t]{3}{*}{$45^{\circ}$} & Present $(10 \times 10)$ & 6.9426 & 11.052 & 15.615 & 16.637 & 20.828 & 22.808 & 26.845 & 29.697 \\
\hline & Present $(11 \times 11)$ & 6.9424 & 11.052 & 15.615 & 16.636 & 20.828 & 22.807 & 26.084 & 29.686 \\
\hline & Ref. [10] & 6.9564 & 11.0782 & 15.6482 & 16.6786 & 20.8790 & 22.9147 & 26.9540 & 30.0230 \\
\hline \multicolumn{10}{|c|}{$\left(90^{\circ} / 0^{\circ} / 90^{\circ} / 0^{\circ}\right)$} \\
\hline \multirow[t]{3}{*}{$30^{\circ}$} & Present $(10 \times 10)$ & 4.8604 & 8.6618 & 11.102 & 12.512 & 17.091 & 17.290 & 20.270 & 22.656 \\
\hline & Present $(11 \times 11)$ & 4.8604 & 8.6618 & 11.102 & 12.511 & 17.091 & 17.290 & 20.270 & 22.656 \\
\hline & Ref. [10] & 4.8701 & 8.6801 & 11.1241 & 12.5388 & 17.1424 & 17.3294 & 20.2977 & 22.7235 \\
\hline \multirow[t]{3}{*}{$45^{\circ}$} & Present $(10 \times 10)$ & 6.9429 & 11.051 & 15.615 & 16.640 & 20.827 & 22.806 & 26.844 & 29.697 \\
\hline & Present $(11 \times 11)$ & 6.9427 & 11.051 & 15.615 & 16.638 & 20.827 & 22.805 & 26.842 & 29.686 \\
\hline & Ref. [10] & 6.9564 & 11.0782 & 15.6482 & 16.6786 & 20.8790 & 22.9147 & 26.9540 & 30.0230 \\
\hline
\end{tabular}

graphite/epoxy material. To have better insight about the effect of different fibre orientation angle $(\beta)$ and number of layers $\left(N_{c}\right)$ on the dynamic properties of these general rhomboidal $(b / a=1)$ antisymmetric angle-ply laminated plates, the variations of the first two free vibration coefficients $\left(\Omega_{1}\right.$ and $\Omega_{2}$ ) are plotted in Figs. 3 and 4 . Two boundary conditions have been included, $\mathrm{S}_{3} \mathrm{~S}_{3} \mathrm{~S}_{3} \mathrm{~S}_{3}$ in Fig. 3 and
$\mathrm{F}_{4} \mathrm{~F}_{4} \mathrm{~F}_{4} \mathrm{C}_{1}$ in Fig. 4. Many others aspect ratios and boundary condition have been studied, but the corresponding results are not included for brevity purposes. In general, it is observed that for all analyzed boundary conditions and aspect ratios, the first two frequency coefficients are minimums for the number of layers $\left(N_{c}\right)$ equal to 2 . When $N_{c} \geqslant 4$, the $\Omega_{i}(i=1,2)$ values are considerably different 

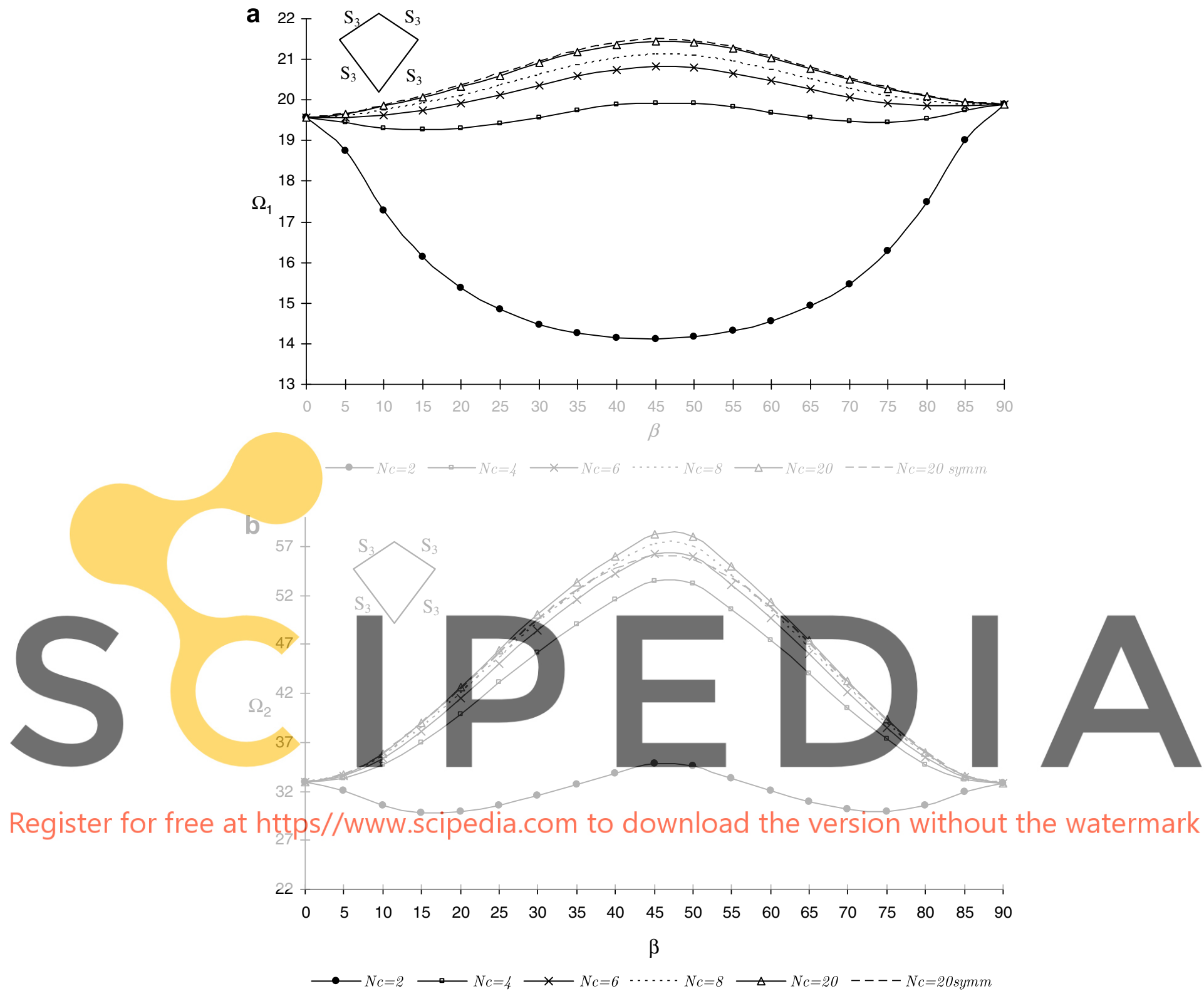

Fig. 3. Effect of fibre orientation and lamination sequence on the first two vibration frequency coefficients for $\mathrm{S}_{3} \mathrm{~S}_{3} \mathrm{~S}_{3} \mathrm{~S}_{3}$ graphite/epoxy rhomboidal plates with $b / a=1$. (a) $\Omega_{1}=\omega_{1} a^{2} \sqrt{\rho h / D_{\beta}}$ versus $\beta$, (b) $\Omega_{2}=\omega_{2} a^{2} \sqrt{\rho h / D_{\beta}}$ versus $\beta$.

from those of $N_{c}=2$. By keeping the total thickness constant, if the number of layers is increased, the frequency coefficients increase. This is due to the reduction in the coupling stiffness terms $B_{16}$ and $B_{26}$. Results for $N_{c}=20$ symmetric lamination were also included in Figs. 3 and 4 and it is observed that, even for many layers, the influence of the stacking sequences is meaningful. Furthermore, it is important to point out that the way of variation of the fundamental frequency is rather different from the way in which varies the frequency that corresponds to the second mode of vibration. This situation occurs for all analyzed boundary conditions and should be specially kept in mind when specific requirements of design that involve at the first or the second vibration modes exist.
Finally, Figs. 5 and 6 show the first five nondimensional free vibration frequencies $\omega a^{2} \sqrt{\rho h / D_{\beta}}$ of arbitrarily laminated graphite/epoxy rhomboidal plates and their corresponding nodal patterns $(b / a=0.5$ in Fig. $5, b / a=1$ in Fig. 6). Three boundary conditions i.e. $S_{1} S_{1} S_{1} S_{1}$, $\mathrm{C}_{1} \mathrm{~F}_{4} \mathrm{~F}_{4} \mathrm{~F}_{4}, \mathrm{C}_{2} \mathrm{~S}_{2} \mathrm{C}_{2} \mathrm{~S}_{2}$ and three stacking sequences i.e. $\left(45^{\circ} \%-45^{\circ}\right),\left(30^{\circ} \%-30^{\circ}\right),\left(0^{\circ} / 45^{\circ}\right)$ are considered in each figure. It is shown that the variation in the frequency coefficients is noticeable for the different stacking sequences, especially between $\left(0^{\circ} / 45^{\circ}\right)$ laminated plates and the antisymmetric angle-ply ones. In arbitrarily laminated plates all the stretching-bending couplings $B_{i j}(i, j=1,2,6)$ are different from zero and it is observed that the frequency parameters are higher in these cases. 


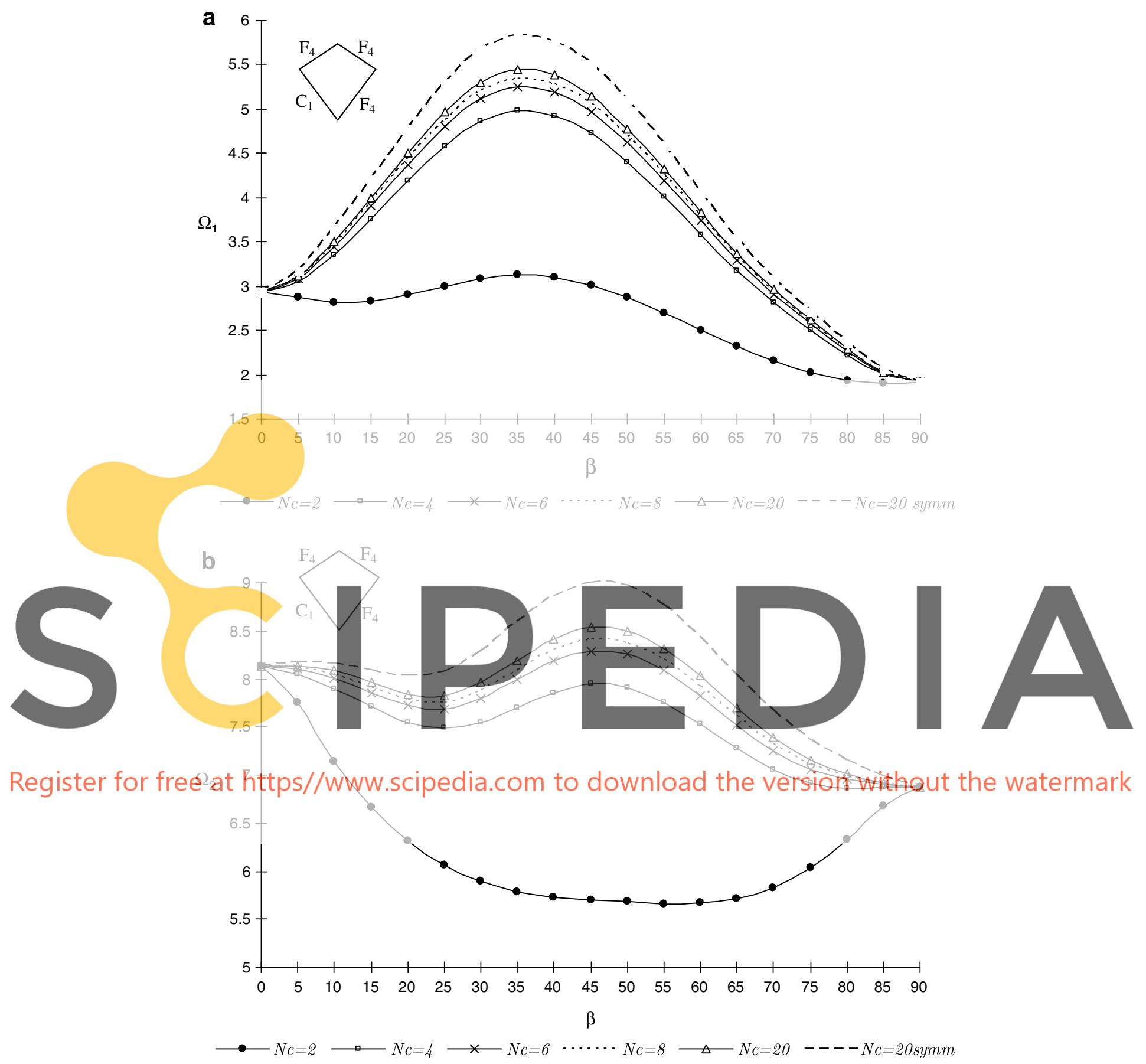

Fig. 4. Effect of fibre orientation and lamination sequence on the first two vibration frequency coefficients for $\mathrm{F}_{4} \mathrm{~F}_{4} \mathrm{~F}_{4} \mathrm{C}_{1}$ graphite/epoxy rhomboidal plates with $b / a=1$. (a) $\Omega_{1}=\omega_{1} a^{2} \sqrt{\rho h / D_{\beta}}$ versus $\beta$, (b) $\Omega_{2}=\omega_{2} a^{2} \sqrt{\rho h / D_{\beta}}$ versus $\beta$.

\section{Concluding remarks}

A simple, computationally efficient and accurate formulation has been developed for the study of the free vibration of arbitrarily laminated composite plates. The methodology is based on the Ritz method and on the classical laminated plate theory, and uses natural coordinates to express the geometry of different laminates in a simple form. The transverse deflection and the in-plane stretching deformation of the plate are approximated by sets of char- acteristic orthogonal polynomials generated using the Gram-Schmidt procedure. The algorithm developed is very general and allows taking into account a great variety of geometrical shapes, material properties and combinations of boundary conditions.

From the convergence studies and the comparisons with results available in the literature it is observed that the approach presented is reliable and accurate. Sets of numerical results are given in tabular and graphical form illustrating the influence of different number of layers, fibre 


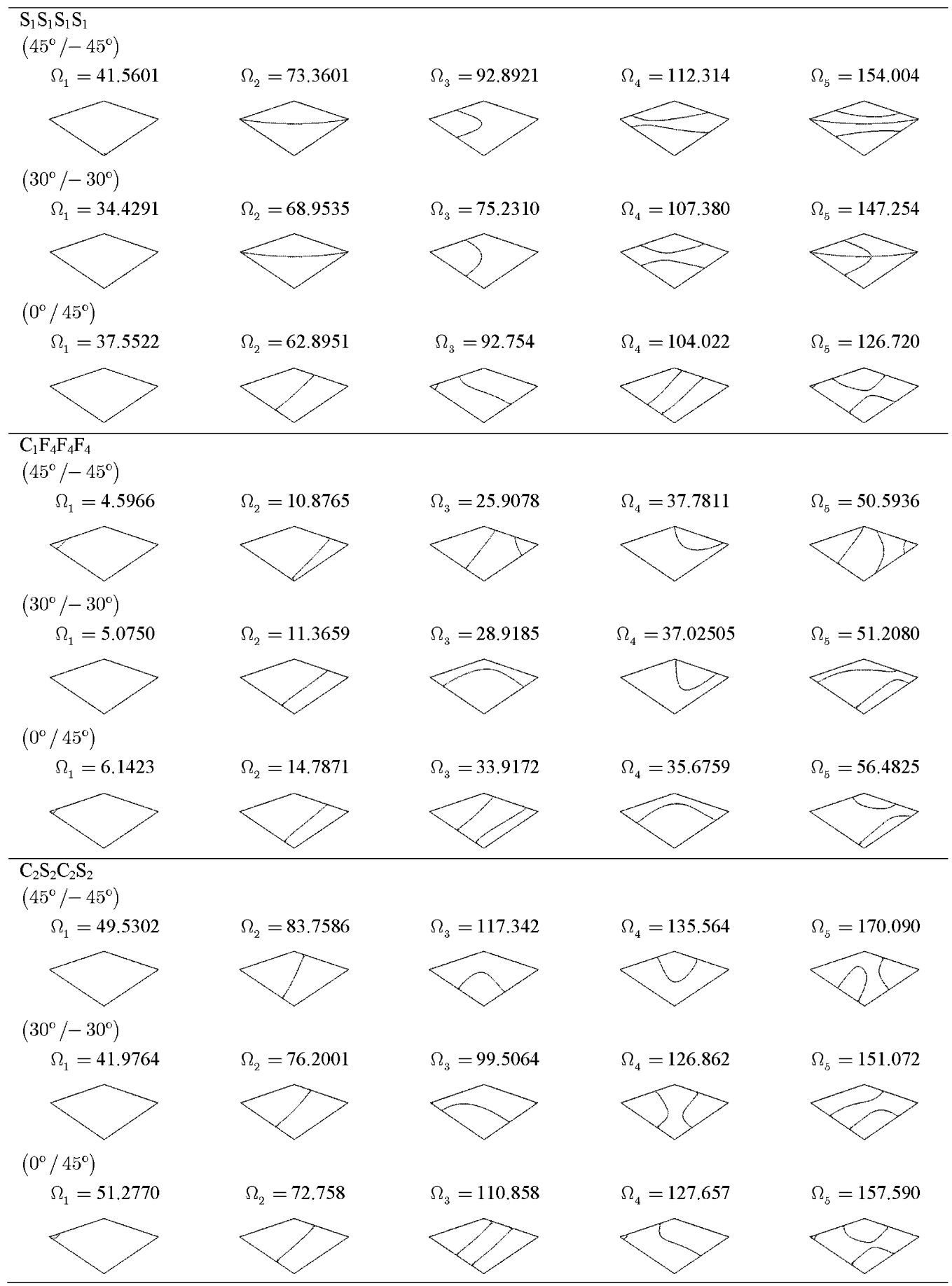

Fig. 5. First five non-dimensional frequency parameters $\Omega_{i}=\omega_{i} a^{2} \sqrt{\rho h / D_{\beta}}(i=1, \ldots, 5)$, modal shapes and nodal patterns for general rhomboidal twolayered plates with $b / a=0.5$.

stacking sequences and edge conditions on the natural frequencies and nodal patterns of a selection of laminated plates. Besides, all applications demonstrate that the present technique is accurate and efficient. Its flexibility offers the clear possibility of varying the parameters involved in the problem in a relatively simple way. Consequently it constitutes an efficient tool for the determination of natural frequencies so much in design problems as in optimization problems. Finally, it is important to point out that the method presented can be easily modified to be applied to static deflection problems.

\section{Appendix A}

Matrices included in the second derivatives of displacement field

The matrices $\left[O p^{(1)}\right]$ and $\left[O p^{(2)}\right]$ that appear in Eq. (6) are as follow: 
322

L. G. Nallim, S. Older/ Composite Structures 85 (2008) 311-325

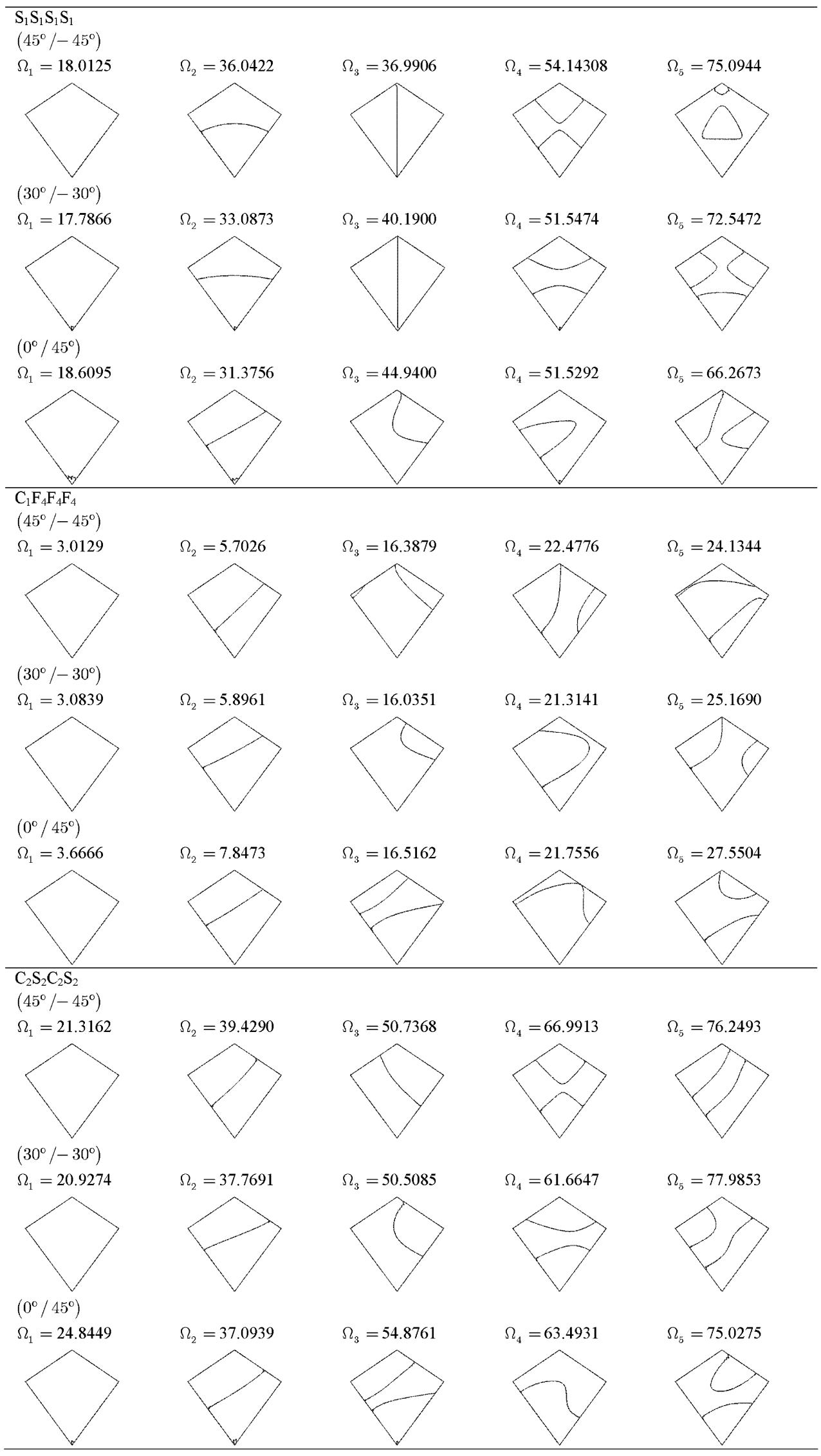

Fig. 6. First five non-dimensional frequency parameters $\Omega_{i}=\omega_{i} a^{2} \sqrt{\rho h / D_{\beta}}(i=1, \ldots, 5)$, modal shapes and nodal patterns for general rhomboidal twolayered plates with $b / a=1$. 


$$
\begin{aligned}
& {\left[O p^{(1)}\right]=\left[\begin{array}{ccc}
a_{1} & a_{2} & -a_{3} \\
b_{1} & b_{2} & -b_{3} \\
-c_{1} & -c_{2} & c_{3}
\end{array}\right],} \\
& {\left[O p^{(2)}\right]=\left[\begin{array}{cc}
\sum_{i=1}^{3} a_{i} \alpha_{i} & \sum_{i=1}^{3} a_{i} \beta_{i} \\
\sum_{i=1}^{3} b_{i} \alpha_{i} & \sum_{i=1}^{3} b_{i} \beta_{i} \\
-\sum_{i=1}^{3} c_{i} \alpha_{i} & -\sum_{i=1}^{3} c_{i} \beta_{i}
\end{array}\right],}
\end{aligned}
$$

where

$a_{1}=J_{22}^{2}, \quad a_{2}=J_{12}^{2}, \quad a_{3}=2 J_{12} J_{22}$

$b_{1}=J_{21}^{2}, \quad b_{2}=J_{11}^{2}, \quad b_{3}=2 J_{11} J_{21}$

$c_{1}=J_{21} J_{22}, \quad c_{2}=J_{11} J_{12}, \quad c_{3}=J_{11} J_{22}+J_{12} J_{21}$

$\alpha_{1}=-J_{11, \xi} J_{22}+J_{12, \xi} J_{21} \quad \alpha_{2}=-J_{21, \eta} J_{22}+J_{22, \eta} J_{21}$,

$\alpha_{3}=J_{11, \eta} J_{22}-J_{22, \xi} J_{21}$

$\beta_{1}=J_{11, \xi} J_{12}-J_{12, \xi} J_{11}, \quad \beta_{2}=J_{21, \eta} J_{12}-J_{22, \eta} J_{11}$,

$\beta_{3}=-J_{11, \eta} J_{12}+J_{22, \xi} J_{11}$

\section{Appendix B}

Functions included in the strain energy

After substitution of Eq. (6) into Eq. (3) one obtains the maximum strain energy as a function of the derivatives of the displacements $U, V$ and $W$ with respect to the natural coordinates $\xi, \eta$. The factors of these derivatives depend on the geometrical and mechanical characteristics of the plates, and are given by

$$
\begin{aligned}
A_{1}^{*}= & A_{11} a_{1}-2 A_{16} c_{1}+A_{66} b_{1} \\
A_{2}^{*}= & A_{11} a_{2}-2 A_{16} c_{2}+A_{66} b_{2} \\
A_{3}^{*}= & -A_{11} a_{3}+2 A_{16} c_{3}-A_{66} b_{3} \\
A_{4}^{*}= & 2\left(A_{12} \sqrt{a_{1} b_{2}}+A_{66} \sqrt{a_{2} b_{1}}\right)-A_{16} a_{3}-A_{26} b_{3} \\
A_{5}^{*}= & -A_{12} a_{3}+2\left(A_{16} a_{1}+A_{26} b_{1}-A_{66} c_{1}\right) \\
A_{6}^{*}= & -2 c_{2}\left(A_{12}+A_{66}\right)+2 A_{16} a_{2}+2 A_{26} b_{2} \\
A_{7}^{*}= & 2\left(A_{12} \sqrt{a_{2} b_{1}}+A_{66} \sqrt{a_{1} b_{2}}\right)-A_{16} a_{3}-A_{26} b_{3} \\
A_{8}^{*}= & A_{22} b_{1}-2 A_{26} c_{1}+A_{66} a_{1} \\
A_{9}^{*}= & A_{22} b_{2}-2 A_{26} c_{2}+A_{66} a_{2} \\
A_{10}^{*}= & -A_{22} 2 \sqrt{b_{1} b_{2}}+2 A_{26} c_{3}-A_{66} a_{3} \\
B_{1}^{*}= & 2\left(\sqrt{a_{1}}\left(B_{11} a_{1}+B_{12} b_{1}-2 B_{16} c_{1}\right)\right. \\
& \left.+\sqrt{b_{1}}\left(-B_{16} a_{1}-B_{26} b_{1}+2 B_{66} c_{1}\right)\right) \\
B_{2}^{*}= & 2\left(\sqrt{a_{1}}\left(B_{11} a_{2}+B_{12} b_{2}-2 B_{16} c_{2}\right)\right. \\
& \left.+\sqrt{b_{1}}\left(-B_{16} a_{2}-2 B_{26} b_{2}+2 B_{66} c_{2}\right)\right)
\end{aligned}
$$

$$
\begin{aligned}
& B_{3}^{*}=2\left(\sqrt{a_{2}}\left(-B_{11} a_{1}-B_{12} b_{1}+2 B_{16} c_{1}\right)\right. \\
& \left.+\sqrt{b_{2}}\left(B_{16} a_{1}+B_{26} b_{1}-2 B_{66} c_{1}\right)\right) \\
& B_{4}^{*}=2\left(\sqrt{a_{1}}\left(-B_{11} a_{3}-B_{12} b_{3}+2 B_{16} c_{3}\right)+\sqrt{b_{1}}\left(B_{16} a_{3}+B_{26} b_{3}\right.\right. \\
& \left.\left.-2 B_{66} c_{3}\right)\right) \\
& B_{5}^{*}=2\left(\sqrt{a_{2}}\left(-B_{11} a_{2}-B_{12} b_{2}+2 B_{16} c_{2}\right)+\sqrt{b_{2}}\left(B_{16} a_{2}+B_{26} b_{2}\right.\right. \\
& \left.\left.-2 B_{66} c_{2}\right)\right) \\
& B_{6}^{*}=2\left(\sqrt{a_{2}}\left(B_{11} a_{3}+B_{12} b_{3}-2 B_{16} c_{3}\right)+\sqrt{b_{2}}\left(-B_{16} a_{3}-B_{26} b_{3}\right.\right. \\
& \left.\left.+2 B_{66} c_{3}\right)\right) \\
& B_{7}^{*}=2\left(\sqrt{a_{1}}\left(B_{11} \sum_{i=1}^{3} a_{i} \alpha_{i}+B_{12} \sum_{i=1}^{3} b_{i} \alpha_{i}-2 B_{16} \sum_{i=1}^{3} c_{i} \alpha_{i}\right)\right. \\
& \left.+\sqrt{b_{1}}\left(-B_{16} \sum_{i=1}^{3} a_{i} \alpha_{i}-B_{26} \sum_{i=1}^{3} b_{i} \alpha_{i}+2 B_{66} \sum_{i=1}^{3} c_{i} \alpha_{i}\right)\right) \\
& B_{8}^{*}=2\left(\sqrt{a_{1}}\left(B_{11} \sum_{i=1}^{3} a_{i} \beta_{i}+2 B_{12} \sum_{i=1}^{3} b_{i} \beta_{i}-2 B_{16} \sum_{i=1}^{3} c_{i} \beta_{i}\right)\right. \\
& \left.+\sqrt{b_{1}}\left(-B_{16} \sum_{i=1}^{3} a_{i} \beta_{i}-B_{26} \sum_{i=1}^{3} b_{i} \beta_{i}+2 B_{66} \sum_{i=1}^{3} c_{i} \beta_{i}\right)\right) \\
& B_{9}^{*}=2\left(\sqrt{a_{2}}\left(-B_{11} \sum_{i=1}^{3} a_{i} \alpha_{i}-B_{12} \sum_{i=1}^{3} b_{i} \alpha_{i}+2 B_{16} \sum_{i=1}^{3} c_{i} \alpha_{i}\right)\right. \\
& \left.+\sqrt{b_{2}}\left(B_{16} \sum_{i=1}^{3} a_{i} \alpha_{i}+B_{26} \sum_{i=1}^{3} b_{i} \alpha_{i}-2 B_{66} \sum_{i=1}^{3} c_{i} \alpha_{i}\right)\right) \\
& B_{10}^{*}=2\left(\sqrt{a_{2}}\left(-B_{11} \sum_{i=1}^{3} a_{i} \beta_{i}-B_{12} \sum_{i=1}^{3} b_{i} \beta_{i}+2 B_{16} \sum_{i=1}^{3} c_{i} \beta_{i}\right)\right. \\
& \left.+\sqrt{b_{2}}\left(B_{16} \sum_{i=1}^{3} a_{i} \beta_{i}+B_{26} \sum_{i=1}^{3} b_{i} \beta_{i}-2 B_{66} \sum_{i=1}^{3} c_{i} \beta_{i}\right)\right) \\
& B_{11}^{*}=2\left(\sqrt{a_{1}}\left(B_{16} a_{1}+B_{26} b_{1}-2 B_{66} c_{1}\right)+\sqrt{b_{1}}\left(-B_{12} a_{1}-B_{22} b_{1}\right.\right. \\
& \left.\left.+2 B_{26} c_{1}\right)\right) \\
& B_{12}^{*}=2\left(\sqrt{a_{1}}\left(B_{16} a_{2}+B_{26} b_{2}-2 B_{66} c_{2}\right)+\sqrt{b_{1}}\left(-B_{12} a_{2}-B_{22} b_{2}\right.\right. \\
& \left.\left.+2 B_{26} c_{2}\right)\right) \\
& B_{13}^{*}=2\left(\sqrt{a_{2}}\left(-B_{16} a_{1}-B_{26} b_{1}+2 B_{66} c_{1}\right)+\sqrt{b_{2}}\left(B_{12} a_{1}+B_{22} b_{1}\right.\right. \\
& \left.\left.-2 B_{26} c_{1}\right)\right) \\
& B_{14}^{*}=2\left(\sqrt{a_{1}}\left(-B_{16} a_{3}-B_{26} b_{3}+2 B_{66} c_{3}\right)+\sqrt{b_{1}}\left(B_{12} a_{3}+B_{22} b_{3}\right.\right. \\
& \left.\left.-2 B_{26} c_{3}\right)\right) \\
& B_{15}^{*}=2\left(\sqrt{a_{2}}\left(-B_{16} a_{2}-B_{26} b_{2}+2 B_{66} c_{2}\right)+\sqrt{b_{2}}\left(B_{12} a_{2}+B_{22} b_{2}\right.\right. \\
& \left.\left.-2 B_{26} c_{2}\right)\right)
\end{aligned}
$$




$$
\begin{aligned}
& B_{16}^{*}=2\left(\sqrt{a_{2}}\left(B_{16} a_{3}+B_{26} b_{3}-2 B_{66} c_{3}\right)+\sqrt{b_{2}}\left(-B_{12} a_{3}-B_{22} b_{3}\right.\right. \\
& \left.\left.+2 B_{26} c_{3}\right)\right) \\
& B_{17}^{*}=2\left(\sqrt{a_{1}}\left(B_{16} \sum_{i=1}^{3} a_{i} \alpha_{i}+B_{26} \sum_{i=1}^{3} b_{i} \alpha_{i}-2 B_{66} \sum_{i=1}^{3} c_{i} \alpha_{i}\right)\right. \\
& \left.+\sqrt{b_{1}}\left(-B_{12} \sum_{i=1}^{3} a_{i} \alpha_{i}-B_{22} \sum_{i=1}^{3} b_{i} \alpha_{i}+2 B_{26} \sum_{i=1}^{3} c_{i} \alpha_{i}\right)\right) \\
& B_{18}^{*}=2\left(\sqrt{a_{1}}\left(B_{16} \sum_{i=1}^{3} a_{i} \beta_{i}+B_{26} \sum_{i=1}^{3} b_{i} \beta_{i}-2 B_{66} \sum_{i=1}^{3} c_{i} \beta_{i}\right)\right. \\
& \left.+\sqrt{b_{1}}\left(-B_{12} \sum_{i=1}^{3} a_{i} \beta_{i}-B_{22} \sum_{i=1}^{3} b_{i} \beta_{i}+2 B_{26} \sum_{i=1}^{3} c_{i} \beta_{i}\right)\right) \\
& B_{19}^{*}=2\left(\sqrt{a_{2}}\left(-B_{16} \sum_{i=1}^{3} a_{i} \alpha_{i}-B_{26} \sum_{i=1}^{3} b_{i} \alpha_{i}+2 B_{66} \sum_{i=1}^{3} c_{i} \alpha_{i}\right)\right. \\
& \left.+\sqrt{b_{2}}\left(B_{12} \sum_{i=1}^{3} a_{i} \alpha_{i}+B_{22} \sum_{i=1}^{3} b_{i} \alpha_{i}-2 B_{26} \sum_{i=1}^{3} c_{i} \alpha_{i}\right)\right) \\
& B_{20}^{*}=2\left(\sqrt{a_{2}}\left(-B_{16} \sum_{i=1}^{3} a_{i} \beta_{i}-B_{26} \sum_{i=1}^{3} b_{i} \beta_{i}+2 B_{66} \sum_{i=1}^{3} c_{i} \beta_{i}\right)\right. \\
& \left.+\sqrt{b_{2}}\left(B_{12} \sum_{i=1}^{3} a_{i} \beta_{i}+B_{22} \sum_{i=1}^{3} b_{i} \beta_{i}-2 B_{26} \sum_{i=1}^{3} c_{i} \beta_{i}\right)\right) \\
& D_{1}^{*}=D_{11} a_{1}^{2}+D_{22} b_{1}^{2}+2 D_{12} a_{1} b_{1}-4 D_{16} a_{1} c_{1}-4 D_{26} b_{1} c_{1} \\
& +4 D_{66} c_{1}^{2} \\
& D_{2}^{*}=D_{11} a_{2}^{2}+D_{22} b_{2}^{2}+2 D_{12} a_{2} b_{2}-4 D_{16} a_{2} c_{2}-4 D_{26} b_{2} c_{2} \\
& +4 D_{66} c_{2}^{2} \\
& D_{3}^{*}=2\left(D_{11} a_{1} a_{2}+D_{22} b_{1} b_{2}+D_{12}\left(b_{1} a_{2}+b_{2} a_{1}\right)-2 D_{16}\left(c_{2} a_{1}\right.\right. \\
& \left.\left.+c_{1} a_{2}\right)-2 D_{26}\left(b_{2} c_{1}+b_{1} c_{2}\right)+4 D_{66} c_{1} c_{2}\right) \\
& D_{4}^{*}=D_{11} a_{3}^{2}+D_{22} b_{3}^{2}+2 D_{12} a_{3} b_{3}-4 D_{16} a_{3} c_{3}-4 D_{26} b_{3} c_{3} \\
& +4 D_{66} c_{3}^{2} \\
& D_{5}^{*}=2\left(-D_{11} a_{3} a_{1}-D_{22} b_{3} b_{1}-D_{12}\left(b_{1} a_{3}+b_{3} a_{1}\right)+2 D_{16}\left(a_{1} c_{3}\right.\right. \\
& \left.\left.+c_{1} a_{3}\right)+2 D_{26}\left(b_{3} c_{1}+b_{1} c_{3}\right)-4 D_{66} c_{3} c_{1}\right) \\
& D_{6}^{*}=2\left(-D_{11} a_{2} a_{3}-D_{22} b_{3} b_{2}-D_{12}\left(b_{3} a_{2}+b_{2} a_{3}\right)+2 D_{16}\left(c_{3} a_{2}\right.\right. \\
& \left.\left.+c_{2} a_{3}\right)+2 D_{26}\left(b_{3} c_{2}+b_{2} c_{3}\right)-4 D_{66} c_{3} c_{2}\right) \\
& D_{7}^{*}=2\left(D_{11} a_{1} \sum_{i=1}^{3} a_{i} \alpha_{i}+D_{22} b_{1} \sum_{i=1}^{3} b_{i} \alpha_{i}\right. \\
& +D_{12}\left(a_{1} \sum_{i=1}^{3} b_{i} \alpha_{i}+b_{1} \sum_{i=1}^{3} a_{i} \alpha_{i}\right) \\
& -2 D_{16}\left(a_{1} \sum_{i=1}^{3} c_{i} \alpha_{i}+c_{1} \sum_{i=1}^{3} a_{i} \alpha_{i}\right) \\
& \left.-2 D_{26}\left(b_{1} \sum_{i=1}^{3} c_{i} \alpha_{i}+c_{1} \sum_{i=1}^{3} b_{i} \alpha_{i}\right)+4 D_{66} c_{1} \sum_{i=1}^{3} c_{i} \alpha_{i}\right) \\
& D_{8}^{*}=2\left(D_{11} a_{2} \sum_{i=1}^{3} a_{i} \beta_{i}+D_{22} b_{2} \sum_{i=1}^{3} b_{i} \beta_{i}\right. \\
& +D_{12}\left(a_{2} \sum_{i=1}^{3} b_{i} \beta_{i}+b_{2} \sum_{i=1}^{3} a_{i} \beta_{i}\right) \\
& -2 D_{16}\left(a_{2} \sum_{i=1}^{3} c_{i} \beta_{i}+c_{2} \sum_{i=1}^{3} a_{i} \beta_{i}\right) \\
& \left.-2 D_{26}\left(c_{2} \sum_{i=1}^{3} b_{i} \beta_{i}+b_{2} \sum_{i=1}^{3} c_{i} \beta_{i}\right)+4 D_{66} c_{2} \sum_{i=1}^{3} c_{i} \beta_{i}\right) \\
& D_{9}^{*}=2\left(D_{11} a_{1} \sum_{i=1}^{3} a_{i} \beta_{i}+D_{22} b_{1} \sum_{i=1}^{3} b_{i} \beta_{i}\right. \\
& +D_{12}\left(a_{1} \sum_{i=1}^{3} b_{i} \beta_{i}+b_{1} \sum_{i=1}^{3} a_{i} \beta_{i}\right) \\
& -2 D_{16}\left(a_{1} \sum_{i=1}^{3} c_{i} \beta_{i}+c_{1} \sum_{i=1}^{3} a_{i} \beta_{i}\right) \\
& \left.-2 D_{26}\left(c_{1} \sum_{i=1}^{3} b_{i} \beta_{i}+b_{1} \sum_{i=1}^{3} c_{i} \beta_{i}\right)+4 D_{66} c_{1} \sum_{i=1}^{3} c_{i} \beta_{i}\right) \\
& D_{10}^{*}=2\left(D_{11} a_{2} \sum_{i=1}^{3} a_{i} \alpha_{i}+D_{22} b_{2} \sum_{i=1}^{3} b_{i} \alpha_{i}\right. \\
& +D_{12}\left(a_{2} \sum_{i=1}^{3} b_{i} \alpha_{i}+b_{2} \sum_{i=1}^{3} a_{i} \alpha_{i}\right) \\
& -2 D_{16}\left(a_{2} \sum_{i=1}^{3} c_{i} \alpha_{i}+c_{2} \sum_{i=1}^{3} a_{i} \alpha_{i}\right) \\
& \left.-2 D_{26}\left(c_{2} \sum_{i=1}^{3} b_{i} \alpha_{i}+b_{2} \sum_{i=1}^{3} c_{i} \alpha_{i}\right)+4 D_{66} c_{2} \sum_{i=1}^{3} c_{i} \alpha_{i}\right) \\
& D_{11}^{*}=2\left(-D_{11} a_{3} \sum_{i=1}^{3} a_{i} \alpha_{i}-D_{22} b_{3} \sum_{i=1}^{3} b_{i} \alpha_{i}\right. \\
& -D_{12}\left(a_{3} \sum_{i=1}^{3} b_{i} \alpha_{i}+b_{3} \sum_{i=1}^{3} a_{i} \alpha_{i}\right) \\
& +2 D_{16}\left(a_{3} \sum_{i=1}^{3} c_{i} \alpha_{i}+c_{3} \sum_{i=1}^{3} a_{i} \alpha_{i}\right) \\
& \left.+2 D_{26}\left(c_{3} \sum_{i=1}^{3} b_{i} \alpha_{i}+b_{3} \sum_{i=1}^{3} c_{i} \alpha_{i}\right)-4 D_{66} c_{3} \sum_{i=1}^{3} c_{i} \alpha_{i}\right) \\
& D_{12}^{*}=2\left(-D_{11} a_{3} \sum_{i=1}^{3} a_{i} \beta_{i}-D_{22} b_{3} \sum_{i=1}^{3} b_{i} \beta_{i}\right. \\
& -D_{12}\left(a_{3} \sum_{i=1}^{3} b_{i} \beta_{i}+b_{3} \sum_{i=1}^{3} a_{i} \beta_{i}\right) \\
& +2 D_{16}\left(a_{3} \sum_{i=1}^{3} c_{i} \beta_{i}+c_{3} \sum_{i=1}^{3} a_{i} \beta_{i}\right) \\
& \left.+2 D_{26}\left(c_{3} \sum_{i=1}^{3} b_{i} \beta_{i}+b_{3} \sum_{i=1}^{3} c_{i} \beta_{i}\right)-4 D_{66} c_{3} \sum_{i=1}^{3} c_{i} \beta_{i}\right)
\end{aligned}
$$




$$
\begin{aligned}
D_{13}^{*}= & D_{11}\left(\sum_{i=1}^{3} a_{i} \alpha_{i}\right)^{2}+D_{22}\left(\sum_{i=1}^{3} b_{i} \alpha_{i}\right)^{2}+2 D_{12} \sum_{i=1}^{3} b_{i} \alpha_{i} \\
& \times \sum_{i=1}^{3} a_{i} \alpha_{i}-4 D_{16} \sum_{i=1}^{3} c_{i} \alpha_{i} \sum_{i=1}^{3} a_{i} \alpha_{i}-4 D_{26} \sum_{i=1}^{3} b_{i}^{\prime} \alpha_{i}^{\prime} \\
& \times \sum_{i=1}^{3} c_{i}^{\prime} \alpha_{i}^{\prime}+4 D_{66}\left(\sum_{i=1}^{3} c_{i}^{\prime} \alpha_{i}^{\prime}\right)^{2} \\
D_{14}^{*}= & D_{11}\left(\sum_{i=1}^{3} a_{i} \beta_{i}\right)^{2}+D_{22}\left(\sum_{i=1}^{3} b_{i} \beta_{i}\right)^{2}+2 D_{12} \sum_{i=1}^{3} b_{i} \beta_{i} \\
& \times \sum_{i=1}^{3} a_{i} \beta_{i}-4 D_{16} \sum_{i=1}^{3} c_{i} \beta_{i} \sum_{i=1}^{3} a_{i} \beta_{i}-4 D_{26} \sum_{i=1}^{3} b_{i} \beta_{i} \\
& \times \sum_{i=1}^{3} c_{i} \beta_{i}+4 D_{66}\left(\sum_{i=1}^{3} c_{i} \beta_{i}\right)^{2} \\
D_{15}^{*}= & 2\left(D_{11} \sum_{i=1}^{3} a_{i} \alpha_{i} \sum_{i=1}^{3} a_{i} \beta_{i}+D_{22} \sum_{i=1}^{3} b_{i} \alpha_{i} \sum_{i=1}^{3} b_{i} \beta_{i}\right. \\
& +D_{12}\left(\sum_{i=1}^{3} b_{i} \beta_{i} \sum_{i=1}^{3} a_{i} \alpha_{i}+\sum_{i=1}^{3} b_{i} \alpha_{i} \sum_{i=1}^{3} a_{i} \beta_{i}\right) \\
& -2 D_{16}\left(\sum_{i=1}^{3} c_{i} \beta_{i} \sum_{i=1}^{3} a_{i} \alpha_{i}+\sum_{i=1}^{3} c_{i} \alpha_{i} \sum_{i=1}^{3} a_{i} \beta_{i}\right) \\
& -2 D_{26}\left(\sum_{i=1}^{3} b_{i} \beta_{i} \sum_{i=1}^{3} c_{i} \alpha_{i}+\sum_{i=1}^{3} b_{i} \alpha_{i} \sum_{i=1}^{3} c_{i=1}^{3} c_{i} \beta_{i} \sum_{i=1}^{3} c_{i} \beta_{i}\right)
\end{aligned}
$$

where $A_{i j}, B_{i j}, D_{i j}(i, j=1,2,6)$ are the conventional laminate stiffness coefficients and $a_{i}, b_{i}, c_{i}, \alpha_{i}, \beta_{i},(i=1, \ldots, 3)$ are defined in Appendix A.

\section{References}

[1] Reddy JN. Mechanics of laminated anisotropic plates: theory and analysis. Boca Raton, FL: CRC Press; 1997.

[2] Baharlou B, Leissa AW. Vibration and buckling of generally laminated composite plates with arbitrary edge conditions. Int $\mathbf{J}$ Mech Sci 1987;29:545-55.

[3] Farsa J, Kukreti AR, Bert CW. Fundamental frequency analysis of laminated rectangular plates by differential quadrature method. Int $\mathbf{J}$ Numer Methods Eng 1993;36:2341-56.

[4] Barton O, Reiss R. Vibration of antisymmetric laminated plates using eigensensitivity analysis. Comput Struct 1997;64:425-39.

[5] Kabir HRH, Al-Khaleefi AM, Chaudhuri RA. Free vibration analysis of thin arbitrarily laminated anisotropic plates using bound- ary-continuous displacement Fourier approach. Compos Struct 2001:53:469-76.

[6] Kabir HRH. On free vibration response and mode shapes of arbitrarily laminated rectangular plates. Compos Struct 2004;65:13-27.

[7] Cheung YK, Zhou D. Free vibrations of rectangular unsymmetrically laminated plates with internal line supports. Comput Struct 2001;79:1923-32.

[8] Liew KM, Lim CW. Vibratory characteristics of general laminates, I: symmetric trapezoids. J Sound Vibr 1995;183:615-42.

[9] Lim CW, Liew KM, Kitipornchai S. Vibration of arbitrarily laminated plates of general trapezoidal planform. J Acoust Soc Am 1996;100:3674-85.

[10] Wang S. Vibration of thin skew fibre reinforced composite laminates. J Sound Vibr 1997;201:335-52.

[11] Krishna Reddy AR, Palaninathan R. Free vibration of skew laminates. Comput Struct 1999;70:415-23.

[12] Nallim LG, Oller S, Grossi RO. Statical and dynamical behaviour of thin fibre reinforced composite laminates with different shapes. Comput Methods Appl Mech Eng 2005;194:1797-822.

[13] Whitney JM. Structural analysis of laminated anisotropic plates. Pennsylvania, USA: Technomic Publishing Co. Inc.; 1987.

[14] Li WY, Cheung YK, Tham LG. Spline finite strip analysis of general plates. J Eng Mech 1986;112:43-54.

[15] Cheung YK, Tham LG, Li WY. Free vibration and static analysis of general plate by spline finite strip method. Comput Mech 1988;3:187-97.

[16] Geannakakes GN. Vibration analysis of arbitrarily shaped plates using beam characteristic orthogonal polynomials in semi-analytical finite strip method. J Sound Vibr 1990;137:283-303.

[17] Wang G, Cheng-Tzu TH. Static and dynamic analysis of arbitrary quadrilateral flexural plates by B3-spline functions. Int $\mathbf{J}$ of Solids Struct 1994;31:657-67.

[18] Bert CW, Malik M. The differential quadrature method for irregular domains and application to plate vibration. Int $\mathrm{J}$ Mech Sci 1996;38:589-606.

[19] Saadatpour MM, Azhari M, Bradford MA. Vibration analysis of simply supported plates of general shape with internal point and line supports using the Galerkin method. Eng Struct 2000;22:1180-8.

[20] Zienkiewicz OC, Taylor RL. The finite element method. 4th ed. New York: McGraw-Hill; 1991.

[21] Jones RM. Mechanics of composite materials. second ed. Philadelphia: Taylor and Francis; 1999.

[22] Rektorys K. Variational methods in mathematics. Dordrecht: Science and Engineering. Reidel Co.; 1980

[23] Kantorovich L, Krylov V. Approximate methods of higher analysis. Interscience Publishers; 1964.

[24] Mikhlin S. Variational methods of mathematical physics. New York: Mac Millan Co.; 1964.

[25] Nallim LG, Grossi RO. On the use of orthogonal polynomials in the study of anisotropic plates. J Sound Vibr 2003;264:1201-7.

[26] Nallim LG, Luccioni BM, Grossi RO. Vibration of general triangular composite plates with elastically restrained edges. Thin Walled Struct 2005;43:1711-45.

[27] Bhat RB. Plate deflection using orthogonal polynomials. J Eng Mech ASCE 1985;101:1301-9.

[28] Bhat RB. Natural frequencies of rectangular plates using characteristic orthogonal polynomials in Rayleigh-Ritz method. J Sound Vibr 1985;102:493-9. 